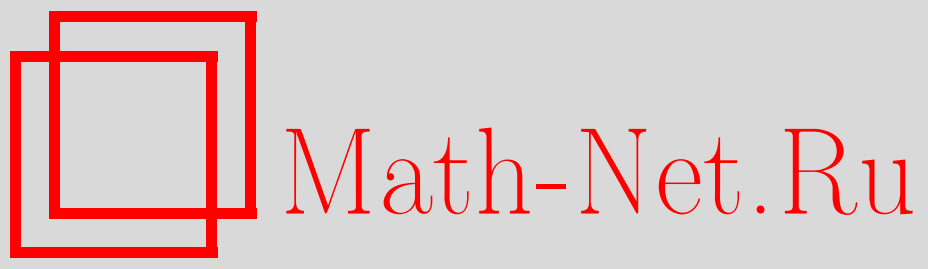

В. Я. Лин, Алгебраические функции, конфигурационные пространства, пространства Тейхмюллера и новые голоморфно-комбинаторные инварианты, Функи. анализ и его прил., 2011, том 45, выпуск $3,55-78$

DOI: https://doi.org/10.4213/faa3040

Использование Общероссийского математического портала MathNet.Ru подразумевает, что вы прочитали и согласны с пользовательским соглашением

http://www . mathnet.ru/rus/agreement

Параметры загрузки:

IP: 3.82 .47 .9

26 апреля 2023 г., 13:06:04

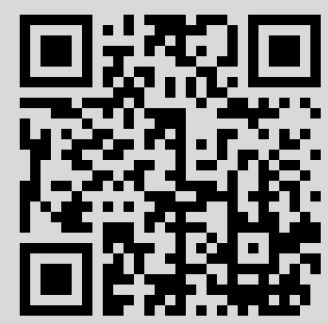




\title{
Алгебраические функции, конфигурационные пространства, пространства Тейхмюллера и новые голоморфно-комбинаторные инварианты
}

\author{
(c) 2011. В. Я. Лин
}

Посвящяется памяти Владимира Игоревича Арнолъда

\begin{abstract}
Доказывается, что при $n \geqslant 4$ функция $u=u_{n}(z), z=\left(z_{1}, \ldots, z_{n}\right) \in \mathbb{C}^{n}$, определяемая уравнением $u^{n}+z_{1} u^{n-1}+\cdots+z_{n}=0$, не может быть ветвью «большей» целой алгебраической функции $g$ на $\mathbb{C}^{n}$, имеющий то же, что $u_{n}$, дискриминантное множество и являющейся суперпозицией целых алгебраических функций от менее чем $n-1$ переменных. Ключевую роль играет описание голоморфных отображений конфигурационных пространств аффинной и проективной прямых $\mathbb{C}$ и $\mathbb{C P}^{1}$, для чего привлекаются пространства Тейхмюллера и новый комбинаторный инвариант комплексных пространств.
\end{abstract}

\section{§1. Введение, основные результаты}

1.1. Суперпозиции; конфигурационные пространства. В 1970 г. В. И. Арнольд [4] доказал, что при $n=2^{r}, r \geqslant 2$, целая $n$-значная алгебраическая функция $u=u_{n}(z), z=\left(z_{1}, \ldots, z_{n}\right) \in \mathbb{C}^{n}$, определяемая уравнением $u^{n}+z_{1} u^{n-1}+\cdots+z_{n}=0$, не является суперпозицией целых алгебраических функций меньшего чем $n-1$ числа переменных. Доказательство основано на том, что (цитирую) «у функций малого числа переменных не бывает многомерных когомологий». В. А. Васильев ([59], [60]) усовершенствовал этот подход, но доставляемые им препятствия срабатывают не для всех $n$. В [40], [42] я нашел другие методы, позволившие распространить теорему Арнольда на все $n>4$.

В упомянутых выше работах речь идет о точных представлениях $u_{n}=g$ : суперпозиции $g$ запрещено иметь лишние ветви, т. е. для всякого $z$ все значения $g(z)$ должны совпадать со значениями $u_{n}(z)$ с учетом кратностей; если лишние ветви у суперпозиции $g$, представляющей $u$, допускаются, то мы пишем $u_{n} \subseteq g$. Одна из целей данной работы - доказательство более сильной теоремы:

Теорема 1. При $n \geqslant 4$ функиия $u_{n}$ не может быть ветвъю целой алгебраической функции $g$, имеющей в $\mathbb{C}^{n}$ в точности то же, что $u_{n}$, дискриминантное множество и являющейся суперпозицией целых алгебраических функций от менее чем $n-1$ переменных.

Это следует из отсутствия точных представлений (§5) и следующей теоремы:

Теорема 2. Если и ивляется ветвъю целой алгебрачческой функиии $g$ на $\mathbb{C}^{n}$ с тем же дискриминантным множеством, что $u u_{n}$, mо $u_{n}=g$.

Конфигурационное пространство $\mathcal{C}^{n}(X)$ связного комплексного пространства $X$ состоит из всех его $n$-точечных подмножеств. Симметрическая группа $\mathbf{S}(n)$ свободно действует на $\mathcal{C}_{o}^{n}(X) \stackrel{\text { def }}{=}\left\{\left(q_{1}, \ldots, q_{n}\right) \in X^{n} \mid q_{i} \neq q_{j}\right.$ для всех $\left.i \neq j\right\}$, 
и накрытие Галуа $p: \mathcal{C}_{o}^{n}(X) \rightarrow \mathcal{C}_{o}^{n}(X) / \mathbf{S}(n)=\mathcal{C}^{n}(X)$ голоморфно. Группы $B_{n}(X)=\pi_{1}\left(\mathcal{C}^{n}(X)\right)$ и $P B_{n}(X)=\pi_{1}\left(\mathcal{C}_{o}^{n}(X)\right) \triangleleft B_{n}(X)$ - это группа кос и группа крашеных кос пространства $X$ соответственно. Всюду в этой статье, если не оговорено противное, $X=\mathbb{C}$ или $\mathbb{C} P^{1}$, а $t(X) \stackrel{\text { def }}{=} \operatorname{dim}_{\mathbb{C}}($ Aut $X)$, так что $t(\mathbb{C})=\operatorname{dim}_{\mathbb{C}} \operatorname{Aff}(\mathbb{C})=2$ и $t\left(\mathbb{C P}^{1}\right)=\operatorname{dim}_{\mathbb{C}} \operatorname{PSL}(2, \mathbb{C})=3$.

Определение 1. Пусть $Z, Z^{\prime} \subseteq \mathbb{C P}^{1}$. Отображение $F: \mathcal{C}^{n}(Z) \rightarrow \mathcal{C}^{m}\left(Z^{\prime}\right)$ назовем зацепляющим ${ }^{1)}$, если $Q \cap F(Q) \neq \varnothing$ для некоторого $Q \in \mathcal{C}^{n}(Z)$ (это условие имеет смысл, так как $Q, F(Q) \subseteq \mathbb{C P}^{1}$ ); в противном случае, т. е. если $Q \cap F(Q)=\varnothing$ для всех $Q \in \mathcal{C}^{n}(Z), F$ называется разделяющим.

Множество ветвления функции $u_{n}$ совпадает с ее дискриминантным множеством, т. е. с множеством нулей $\Sigma_{n}=\left\{z \in \mathbb{C}^{n} \mid d_{n}(z)=0\right\}$ дискриминанта $d_{n}$ ее определяющего полинома. Штейнова область $G_{n}=\mathbb{C}^{n} \backslash \Sigma_{n}$ отождествляется с пространством $\mathbf{G}_{n}$ унитарных полиномов степени $n$ с простыми корнями, которое естественно изоморфно $\mathcal{C}^{n}(\mathbb{C})$.

Пусть $g$ - целая $(n+k)$-значная алгебраическая функция на $\mathbb{C}^{n}$ с тем же, что у $u_{n}$, дискриминантным множеством $\Sigma_{n}$ и такая, что $u_{n} \varsubsetneqq g$. Если $z \in G_{n}$, то $n$-точечное множество $Q_{z}=u_{n}(z)$ является собственным подмножеством $(n+k)$-точечного множества $Q_{z}^{\prime}=g(z)$. Возникает голоморфное отображение $F: \mathcal{C}^{n}(\mathbb{C}) \ni Q_{z} \mapsto Q_{z}^{\prime} \backslash Q_{z} \in \mathcal{C}^{k}(\mathbb{C})$, явлющееся разделяющим; следующая теорема устанавливает отсутствие таковых, обосновывая тем самым теорему 2.

Теорема 3. Пусть $X$ есть $\mathbb{C P}^{1}$ или $\mathbb{C}$. Тогда при $n>t(X)+1 u k \geqslant 1$ всякое голоморфное отображение $F: \mathcal{C}^{n}(X) \rightarrow \mathcal{C}^{k}(X)$ является зацепляюоим.

\section{2. Ручные и одноорбитные морфизмы. Диагональное действие}

$$
\text { Aut } X \ni A: \mathcal{C}^{n}(X) \ni Q=\left\{q_{1}, \ldots, q_{n}\right\} \mapsto\left\{A q_{1}, \ldots, A q_{n}\right\}=A Q \in \mathcal{C}^{n}(X)
$$

в $\mathcal{C}^{n}(X)$ позволяет сопоставить каждому морфизму $T: \mathcal{C}^{n}(X) \rightarrow$ Aut $X$ ручной эндоморфизм $F_{T}$ пространства $\mathcal{C}^{n}(X)$ :

$$
F_{T}: \mathcal{C}^{n}(X) \rightarrow \mathcal{C}^{n}(X), \quad Q \mapsto F_{T}(Q) \stackrel{\text { def }}{=} T(Q) Q \quad \text { для всех } Q \in \mathcal{C}^{n}(X) .
$$

Такой эндоморфизм сохраняет каждую (Aut $X)$-орбиту в $\mathcal{C}^{n}(X)$. С другой стороны, отображение $F: \mathcal{C}^{n}(X) \rightarrow \mathcal{C}^{k}(X)$ назовем одноорбитным, если весь его образ $F\left(\mathcal{C}^{n}(X)\right)$ содержится в одной $($ Aut $X)$-орбите в $\mathcal{C}^{k}(X)$.

Непрерывные отображения линейно связных пространств естественно делятся на абелевы и неабелевы в зависимости от того, абелев или неабелев образ индуцированного данным отображением гомоморфизма фундаментальных групп. Этой очевидной алгебраической дихотомии отвечает нетривиальная аналитическая дихотомия для морфизмов некоторых конфигурационных пространств.

Теорема 4. Пусть $X$ - негиперболическая риманова поверхность, $n>4$ и $F$ - эндоморфизм пространства $\mathcal{C}^{n}(X)$. Тогда $F$ либо ручной, либо одноорбитный; последнее равносильно тому, что $F$ абелев, т.е. образ $F_{*}\left(\pi_{1}\left(\mathcal{C}^{n}(X)\right)\right)$ индуиированного эндоморфизма $F_{*}$ группы кос $B_{n}(X)=\pi_{1}\left(\mathcal{C}^{n}(X)\right)$ абелев.

Эскиз доказательства теоремы 4 для $X=\mathbb{C}$ см. в [39], [43], полное доказательство для $X=\mathbb{C}$ или $\mathbb{C P}^{1}-$ в [50]; лишь эти случаи рассматриваются в

1) В п. 2.3 мы дважды варьируем это определение, приспосабливая его к обстоятельствам. 
данной работе. Для $X=\mathbb{C}^{*}$ эта теорема доказана в [65] (где $\mathcal{C}^{n}\left(\mathbb{C}^{*}\right)$ «притворяется» комплексной камерой Вейля серии $B$ ), а для торов - в [20].

1.3. Примеры. Морфизм Феррари $(1540 \text { г. })^{1)}$ переводит полином степени 4 в его кубическую резольвенту и порождает неабелев морфизм $\mathcal{C}^{4}(\mathbb{C}) \rightarrow \mathcal{C}^{3}(\mathbb{C})$. Менее известен инволютивный автоморфизм Эйзенштейна-Кэли ([18], [13]) пространства невырожденных проективных бинарных форм степени 3, т. е. пространства $\mathcal{C}^{3}\left(\mathbb{C P}^{1}\right)$, сопоставляющий форме $\phi$ якобиан $J(x, y)=\partial(\phi, H \phi) / \partial(x, y)$ ее самой и ее гессиана $H \phi$. (На цитированные работы Эйзенштейна и Кэли, стоящие у истоков теории инвариантов, мне указал М. Х. Гизатуллин.)

В связи с теоремой 3 заметим, что есть разделяющее полиномиальное отображение $F_{3,6}: \mathcal{C}^{3}(\mathbb{C}) \rightarrow \mathcal{C}^{6}(\mathbb{C}), F_{3,6}\left(\left\{q_{1}, q_{2}, q_{3}\right\}\right)=\left\{w_{1}^{+}, w_{1}^{-}, w_{2}^{+}, w_{2}^{-}, w_{3}^{+}, w_{3}^{-}\right\}$, где 6 точек $w_{i}^{ \pm}$- это корни квадратных уравнений $\left(w_{1}^{ \pm}-q_{1}\right)^{2}=\left(q_{1}-q_{2}\right)\left(q_{1}-q_{3}\right)$, $\left(w_{2}^{ \pm}-q_{2}\right)^{2}=\left(q_{2}-q_{3}\right)\left(q_{2}-q_{1}\right),\left(w_{3}^{ \pm}-q_{3}\right)^{2}=\left(q_{3}-q_{1}\right)\left(q_{3}-q_{2}\right)$ (см. [45]).

Структура статьи. В $\S 2$, используя отсутствие голоморфных сечений проколотого универсального семейства Тейхмюллера ([25], [16], [26], [17]), мы доказываем теорему 3, а затем теоремы 2 и 1 ; для последней нужно также отсутствие точных представлений, доказываемое в §5 с помощью теоремы 4.

Главное в $\S 3$ - теорема 10 о сохранении подгруппы $P B_{n}(X)$ неабелевыми эндоморфизмами группы $B_{n}(X)$, гарантирующая подъем неабелевых эндоморфизмов пространства $\mathcal{C}^{n}(X)$ на $\mathcal{C}_{o}^{n}(X)$; ее доказательство основано на описании неабелевых гомоморфизмов $B_{n}(X) \rightarrow \mathbf{S}(k)$, начатом $Э$. Артином [10] и продолженном в [38], [41], [43], [46], [49] и в данной работе (теорема 8).

Теорема 4 доказана в $\$ 4$. Благодаря теореме 10 неабелевы эндоморфизмы пространства $\mathcal{C}^{n}(X)$ накрываются $\mathbf{S}(n)$-эквивариантными эндоморфизмами пространства $\mathcal{C}_{o}^{n}(X)$. Для их описания в п. 4.4 вводится новый инвариант, сопоставляющий (при выбранной заранее комплексной группе Ли $G$; в данной работе $G=\mathbb{C}^{*}$ ) комплексному пространству $Z$ симплициальный комплекс $\mathscr{C}(Z, G)$, вершины которого - все непостоянные голоморфные отображения $Z \rightarrow G \backslash\{1\}$, а симплексы - такие множества вершин $\left\{\mu_{0}, \ldots, \mu_{m}\right\}$, что все $\mu_{i} \cdot \mu_{j}^{-1}(i \neq j)$ - тоже вершины. Исследовав комплекс $\mathscr{C}\left(\mathcal{C}_{o}^{n}(X), \mathbb{C}^{*}\right)$ и естественное $\mathbf{S}(n)$-действие на нем (пп. 4.3, 4.5 и 4.6), мы превращаем исходную аналитическую задачу в комбинаторную, которая и решается в п. 4.7.

\section{§2. Доказательство теоремы 3}

\section{1. Универсальное накрытие $\tau: \mathrm{T}(0, m+3) \rightarrow \mathcal{C}_{o}^{m}(\mathbb{C} \backslash\{0,1\})$. То-} тальное пространство $\mathbf{V}^{\prime}(0, n)$ проколотого универсального семейства Тейхмюллера $\rho: \mathbf{V}^{\prime}(0, n) \rightarrow \mathbf{T}(0, n)$ над пространством Тейхмюллера $\mathbf{T}(0, n)$ римановой сферы с $n$ проколами можно реализовать как дополнение в $\mathbb{C P}^{1} \times \mathbf{T}(0, n)$ к объединению графиков $n$ попарно не пересекающихся голоморфных сечений $\mathbf{s}_{i}: \mathbf{T}(0, n) \rightarrow \mathbb{C P}^{1} \times \mathbf{T}(0, n)$, три последние из которых тождественно равны 0 , 1 и $\infty$. По теореме Хаббарда-Эрла-Kра ([25], [16], [26], [17]) при $\operatorname{dim}_{\mathbb{C}} \mathbf{T}(0, n)=$ $n-3>1$ проекция $\rho$ не имеет голоморфных сечений $\mathbf{T}(0, n) \rightarrow \mathbf{V}^{\prime}(0, n)$.

1) Метод Феррари, сводящий решение уравнения степени 4 к задаче нахождения корней вспомогательного кубического уравнения, решенной чуть ранее Тартальей, опубликован впервые в книге Кардано «Artis Magnæ» (1545г.). Дискриминант резольвенты полинома $F p$ равен квадрату дискриминанта исходного полинома $p$; поэтому корни полинома $F p$ просты, если таковы корни полинома $p$. 
Пусть $n=m+3$ и $\widetilde{\mathbf{s}}_{i}(\mathbf{t})(\mathbf{t} \in \mathbf{T}(0, m+3))$ - проекция точки $\mathbf{s}_{i}(\mathbf{t})$ на $\mathbb{C P}^{1}$; отображение $\tau: \mathbf{T}(0, m+3) \ni \mathbf{t} \mapsto\left(\widetilde{\mathbf{s}}_{1}(\mathbf{t}), \ldots, \widetilde{\mathbf{s}}_{m}(\mathbf{t})\right) \in \mathcal{C}_{o}^{m}(\mathbb{C} \backslash\{0,1\})$ является неразветвленным голоморфным накрытием пространства $\mathcal{C}_{o}^{m}(\mathbb{C} \backslash\{0,1\})$ упорядоченных $m$-точечных конфигураций $\left\{z_{1}, \ldots, z_{m}\right\} \subset \mathbb{C} \backslash\{0,1\}$. Это накрытие универсально, ибо $\mathbf{T}(0, m+3)$ гомеоморфно $2 m$-мерному шару. ${ }^{1)}$

2.2. Отсутствие многолистных сечений. Пусть $\pi$ - расслоение «забывания» первой координаты в $\mathcal{C}_{o}^{m+1}(\mathbb{C} \backslash\{0,1\})$ :

$$
\pi: \mathcal{C}_{o}^{m+1}(\mathbb{C} \backslash\{0,1\}) \ni\left(\zeta, z_{1}, \ldots, z_{m}\right)=(\zeta, z) \mapsto z \in \mathcal{C}_{o}^{m}(\mathbb{C} \backslash\{0,1\}) .
$$

Лемма 1. Морфизмы $\rho, \tau$ и $\pi$ включаются в коммутативную диаграмму

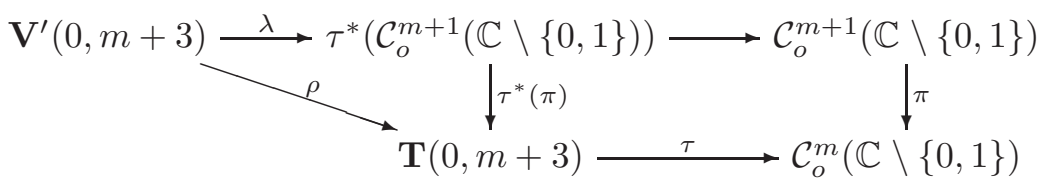

в которой $\tau^{*}(\pi)$ - расслоение, индуцированное расслоением $\pi$ при отображении баз $\tau$, а $\lambda$ - послойный биголоморфный изоморфизм.

Доказательство. Точка $\mathbf{v} \in \mathbf{V}^{\prime}(0, m+3)$ однозначно представляется в виде пары $(\zeta, \mathbf{t})=(\zeta, \rho(\mathbf{v})) \in(\mathbb{C} \backslash\{0,1\}) \times \mathbf{T}(0, m+3)$ с $\zeta \notin\left\{\widetilde{\mathbf{s}}_{1}(\mathbf{t}), \ldots, \widetilde{\mathbf{s}}_{m}(\mathbf{t})\right\}$. Формулы

$$
\begin{aligned}
\lambda: \mathbf{V}^{\prime}(0, m+3) \ni \mathbf{v} & =(\zeta, \rho(\mathbf{v})) \mapsto\left(\mathbf{t}, \zeta, \widetilde{\mathbf{s}}_{1}(\mathbf{t}), \ldots, \widetilde{\mathbf{s}}_{m}(\mathbf{t})\right) \\
& \stackrel{\text { def }}{=}\left(\rho(\mathbf{v}), \zeta, \widetilde{\mathbf{s}}_{1}(\rho(\mathbf{v})), \ldots, \widetilde{\mathbf{s}}_{m}(\rho(\mathbf{v}))\right) \in \tau^{*}\left(\mathcal{C}_{o}^{m+1}(\mathbb{C} \backslash\{0,1\})\right)
\end{aligned}
$$

И

$$
\lambda^{-1}: \tau^{*}\left(\mathcal{C}_{o}^{m+1}(\mathbb{C} \backslash\{0,1\})\right) \ni(\mathbf{t},(\zeta, z)) \mapsto(\zeta, \mathbf{t}) \in \mathbf{V}^{\prime}(0, m+3)
$$

определяют взаимно обратные послойные голоморфные отображения.

Следующая теорема - ключ к доказательству теоремы 3.

Теорема 5. Пусть $m>1$ и $A \subset \mathcal{C}_{o}^{m+1}(\mathbb{C} \backslash\{0,1\})$ - аналитическое подмножество. Тогда сужение на $A$ проекиии $(3),\left.\pi\right|_{A}: A \ni(\zeta, z) \mapsto z \in \mathcal{C}_{o}^{m}(\mathbb{C} \backslash\{0,1\})$, не может быть неразветвленным накрытием всего $\mathcal{C}_{o}^{m}(\mathbb{C} \backslash\{0,1\})$.

Доказательство. Предположим противное. Тогда отображение $\tau$ индуцирует неразветвленное накрытие $\tau^{*}\left(\left.\pi\right|_{A}\right): \tau^{*}(A) \rightarrow \mathbf{T}(0, m+3)$, которое ввиду стягиваемости базы тривиально и, стало быть, допускает голоморфное сечение $s: \mathbf{T}(0, m+3) \rightarrow \tau^{*}(A) \subset \tau^{*}\left(\mathcal{C}_{o}^{m+1}(\mathbb{C} \backslash\{0,1\})\right)$. Композиция

$$
\lambda^{-1} \circ s: \mathbf{T}(0, m+3) \stackrel{s}{\longrightarrow} \tau^{*}(A) \subset \tau^{*}\left(\mathcal{C}_{o}^{m+1}(\mathbb{C} \backslash\{0,1\})\right) \stackrel{\lambda^{-1}}{\longrightarrow} \mathbf{V}^{\prime}(0, m+3)
$$

1) $\mathrm{B}[28],[30]$, [32], [11] при построении универсального накрытия пространства $\mathcal{C}_{o}^{m}(\mathbb{C} \backslash$ $\{0,1\})$ используются квазиконформные отображения; здесь я обхожу их, оперируя непосредственно универсальным семейством Тейхмюллера, которое все равно понадобится в дальнейшем. 
задает голоморфное сечение универсального семейства Тейхмюлера; так как $\operatorname{dim}_{\mathbb{C}} \mathbf{T}(0, m+3)=m>1$, это противоречит теореме Хаббарда-Эрла-Кра.

2.3. Зацепляющие отображения; доказательства теорем 3, 2 и 1. Пусть $X=\mathbb{C}$ или $\mathbb{C P}^{1}$. Отображение $\phi: \mathcal{C}_{o}^{m}(\mathbb{C} \backslash\{0,1\}) \rightarrow \mathcal{C}^{k}(X)$ назовем зацепляющим, если найдется такая точка $z=\left(z_{1}, \ldots, z_{m}\right) \in \mathcal{C}_{o}^{m}(\mathbb{C} \backslash\{0,1\})$, что $\left\{z_{1}, \ldots, z_{m}, 0,1, \infty\right\} \cap \phi(z) \neq \varnothing$.

Теорема 6. При $m>1$ u $k \geqslant 1$ каждий морфизм $\phi: \mathcal{C}_{o}^{m}(\mathbb{C} \backslash\{0,1\}) \rightarrow \mathcal{C}^{k}(X)$ является зацепляющим.

Доказательство. Если для всех $z=\left(z_{1}, \ldots, z_{m}\right) \in \mathcal{C}_{o}^{m}(\mathbb{C} \backslash\{0,1\})$ пересечение $\left\{z_{1}, \ldots, z_{m}, 0,1, \infty\right\} \cap \phi(z)$ пусто, то $\phi(z) \subset \mathbb{C}$ при любом $z$, ибо $\infty \notin \phi(z)$. Пусть $A \stackrel{\text { def }}{=}\left\{(t, z) \in \mathbb{C} \times \mathcal{C}_{o}^{m}(\mathbb{C} \backslash\{0,1\}) \mid t \in \phi(z)\right\}$; тогда $A-$ аналитическое подмножество в $\mathcal{C}_{o}^{m}(\mathbb{C} \backslash\{0,1\})$, и сужение на $A$ проекции $(3)$ является неразветвленным голоморфным накрытием всего $\mathcal{C}_{o}^{m}(\mathbb{C} \backslash\{0,1\})$, что противоречит теореме 5 .

Следствие 1. Пусть $X=\mathbb{C P}^{1}$ или $\mathbb{C}$. Тогда при $n>t(X)+1$ u $k \geqslant 1$ каждое голоморфное отображсние $g: \mathcal{C}_{o}^{n}(X) \rightarrow \mathcal{C}^{k}(X)$ является зацепляющим, m.е. найдется точка $q=\left(q_{1}, \ldots, q_{n}\right) \in \mathcal{C}_{o}^{n}(X)$, для которой $p(q) \cap g(q) \neq \varnothing$ (здесъ, как обычно, $p: \mathcal{C}_{o}^{n}(X) \rightarrow \mathcal{C}^{n}(X)$ - стандартная проекиия).

Доказательство. Пусть $m=n-t(X)$ и $i: \mathcal{C}_{o}^{m}(\mathbb{C} \backslash\{0,1\}) \hookrightarrow \mathcal{C}_{o}^{n}(X)-$ естественное вложение, т. е. $i\left(z_{1}, \ldots, z_{m}\right)=\left(z_{1}, \ldots, z_{m}, 0,1, \infty\right)$ при $X=\mathbb{C P}^{1}$ и $i\left(z_{1}, \ldots, z_{m}\right)=\left(z_{1}, \ldots, z_{m}, 0,1\right)$ при $X=\mathbb{C}$. Применение теоремы 6 к морфизму $\phi=g \circ i: \mathcal{C}_{o}^{m}(\mathbb{C} \backslash\{0,1\}) \underset{i}{\hookrightarrow} \mathcal{C}_{o}^{n}(X) \underset{g}{\rightarrow} \mathcal{C}^{k}(X)$ завершает доказательство.

Доказательство теорем 3, 2 и 1. Согласно следствию 1, отображение $F \circ p: \mathcal{C}_{o}^{n}(X) \stackrel{p}{\rightarrow} \mathcal{C}^{n}(X) \stackrel{F}{\rightarrow} \mathcal{C}^{k}(X)$ является зацепляющим; значит, таков и сам морфизм $F$. Теорема 3 влечет за собой теорему 2 , из которой в силу невозможности точного представления (см. §5) следует теорема 1.

\section{§3. Гомоморфизмы групп кос}

Главное в этом параграфе - теорема 10, гарантирующая подъем на $\mathcal{C}_{o}^{n}(X)$ всех неабелевых эндоморфизмов пространства $\mathcal{C}^{n}(X)\left(X=\mathbb{C}\right.$ или $\left.\mathbb{C P}^{1}\right)$.

3.1. Группы кос $B_{n}=\pi_{1}\left(\mathcal{C}^{n}(\mathbb{C})\right)$ и $B_{n}\left(S^{2}\right)=\pi_{1}\left(\mathcal{C}^{n}\left(\mathbb{C P}^{1}\right)\right)$. Каноническое копредставление группы $B_{n}$ : образующие $\sigma_{1}, \ldots, \sigma_{n-1}$, соотношения $\sigma_{i} \sigma_{j}=$ $\sigma_{j} \sigma_{i}$ при $|i-j|>1$ и $\sigma_{i} \sigma_{i+1} \sigma_{i}=\sigma_{i+1} \sigma_{i} \sigma_{i+1}$ при $1 \leqslant i \leqslant n-2$. Далее, $B_{n}\left(S^{2}\right)=$ $B_{n} / N$, где $N \triangleleft B_{n}$ порождается элементом $\nu=\sigma_{1} \cdots \sigma_{n-1} \sigma_{n-1} \cdots \sigma_{1} \in P B_{n}$.

3.2. Универсальные накрытия, гомотопические группы. Пусть $n \geqslant$ $t(X)=\operatorname{dim}_{\mathbb{C}}($ Aut $X), \mathcal{D}^{n-2}(\mathbb{C})=\left\{q=\left(q_{1}, \ldots, q_{n}\right) \in \mathcal{C}_{o}^{n}(\mathbb{C}) \mid q_{n-1}=0, q_{n}=1\right\}$ и $\mathcal{D}^{n-3}\left(\mathbb{C P}^{1}\right)=\left\{q=\left(q_{1}, \ldots, q_{n}\right) \in \mathcal{C}_{o}^{n}\left(\mathbb{C P}^{1}\right) \mid q_{n-2}=0, q_{n-1}=1, q_{n}=\infty\right\}$. Конечно, $\mathcal{D}^{n-t(X)}(X) \cong \mathcal{C}_{o}^{n-t(X)}(\mathbb{C} \backslash\{0,1\})$. Группа Aut $X$ действует в $\mathcal{C}_{o}^{n}(X)$ свободно и бирегулярно; каждая ее орбита пересекает $\mathcal{D}^{n-t(X)}(X)$ в единственной точке, что дает бирегулярный изоморфизм $j_{X, n}: \mathcal{C}_{o}^{n}(X) \cong($ Aut $X) \times \mathcal{C}_{o}^{n-t(X)}(\mathbb{C} \backslash\{0,1\})$.

Пусть $\mathbb{C} 入 \mathbb{C}$ - полупрямое произведение групп с умножением $(b, \zeta) \cdot\left(b^{\prime}, \zeta^{\prime}\right)=$ $\left(b+e^{\zeta} b^{\prime}, \zeta+\zeta^{\prime}\right)$ и комплексной структурой $\mathbb{C} \times \mathbb{C}$. Голоморфные универсальные 
накрытия $\vartheta_{X}: \widehat{\operatorname{Aut} X} \rightarrow \operatorname{Aut} X$ и $\widehat{\mathcal{C}_{o}^{n}(X)} \rightarrow \mathcal{C}_{o}^{n}(X) \rightarrow \mathcal{C}^{n}(X)$ таковы:

$$
\begin{aligned}
\vartheta_{\mathbb{C}} & : \mathbb{C} \lambda \mathbb{C} \ni(b, \zeta) \mapsto \vartheta_{\mathbb{C}}(b, \zeta)=A \in \mathbf{A f f} \mathbb{C}, \quad A z=e^{\zeta} z+b \forall z \in \mathbb{C}, \\
\vartheta_{\mathbb{C P}^{1}} & : \mathbf{S L}(2, \mathbb{C}) \rightarrow \mathbf{S L}(2, \mathbb{C}) /\{ \pm I\}=\mathbf{P S L}(2, \mathbb{C})=\operatorname{Aut} \mathbb{C P}^{1},
\end{aligned}
$$

$\widehat{\operatorname{Aut} X} \times \mathbf{T}(0, n+3-t(X)) \ni(A, \mathbf{t}) \stackrel{\vartheta}{\mapsto}\left(\vartheta_{X}(A) \widetilde{\mathbf{s}}_{1}(\mathbf{t}), \ldots, \vartheta_{X}(A) \widetilde{\mathbf{s}}_{n}(\mathbf{t})\right) \in \mathcal{C}_{o}^{n}(X)$,

$\widehat{\text { Aut } X} \times \mathbf{T}(0, n+3-t(X)) \ni(A, \mathbf{t}) \stackrel{\Theta}{\mapsto}\left\{\vartheta_{X}(A) \widetilde{\mathbf{s}}_{1}(\mathbf{t}), \ldots, \vartheta_{X}(A) \widetilde{\mathbf{s}}_{n}(\mathbf{t})\right\} \in \mathcal{C}^{n}(X)$

(определение отображений $\widetilde{\mathbf{s}}_{i}$ см. в п. 2.1). Наконец, ввиду изоморфизма $j_{X, n}$

$$
P B_{n}(X)=\pi_{1}\left(\mathcal{C}_{o}^{n}(X)\right)= \begin{cases}\mathbb{Z} \times \pi_{1}\left(\mathcal{C}_{o}^{n-2}(\mathbb{C} \backslash\{0,1\})\right) & \text { при } X=\mathbb{C}, \\ (\mathbb{Z} / 2 \mathbb{Z}) \times \pi_{1}\left(\mathcal{C}_{o}^{n-3}(\mathbb{C} \backslash\{0,1\})\right) & \text { при } X=\mathbb{C P}^{1} .\end{cases}
$$

Следующие результаты являются классическими ([8], [51], [19]): $\mathcal{C}_{o}^{n}(\mathbb{C}) u \mathcal{C}^{n}(\mathbb{C})$ являются $K(\pi, 1)$-пространствами для $\pi=P B_{n} u \pi=B_{n}$ соответственно. Все $\mathcal{C}_{o}^{m}(\mathbb{C} \backslash\{0, \ldots, k-1\})$ асферичны. $B$ группе $P B_{n}$ есть конечный ряд нормальных подгрупп

$$
\{1\}=P B_{n}^{(n)} \subset P B_{n}^{(n-1)} \subset \cdots \subset P B_{n}^{(2)} \subset P B_{n}^{(1)}=P B_{n}
$$

со свободньми факторами $P B_{n}^{(r)} / P B_{n}^{(r+1)} \cong \mathbb{F}_{r}, \operatorname{rank} \mathbb{F}_{r}=r, 1 \leqslant r \leqslant n-1$.

Заменяя в $(7)$ и $(8) n$ на $n-1$, получим $\pi_{1}\left(\mathcal{C}_{o}^{n-3}(\mathbb{C} \backslash\{0,1\})\right)=P B_{n-1}^{(2)}$; вместе с (6) и асферичностью пространства $\mathcal{C}_{o}^{n-3}(\mathbb{C} \backslash\{0,1\})$ это дает следующую теорему (cM. [48], [50]):

Теорема 7. $P B_{3}\left(S^{2}\right) \cong \mathbb{Z} / 2 \mathbb{Z}$. Ecли $n>3$, mo $P B_{n}\left(S^{2}\right) \cong(\mathbb{Z} / 2 \mathbb{Z}) \times P B_{n-1}^{(2)}$, причем группа $P B_{n-1}^{(2)}$ обладает конечньм рядом нормальных подгрупп

$$
\{1\}=P B_{n-1}^{(n-1)} \subset P B_{n-1}^{(n-2)} \subset \cdots \subset P B_{n-1}^{(3)} \subset P B_{n-1}^{(2)}
$$

со свободными бакторами $P B_{n-1}^{(r)} / P B_{n-1}^{(r+1)} \cong \mathbb{F}_{r}, 2 \leqslant r \leqslant n-2$. При $n \geqslant 3$ имеeм $\pi_{k}\left(\mathcal{C}^{n}\left(\mathbb{C P}^{1}\right)\right)=\pi_{k}\left(\mathcal{C}_{o}^{n}\left(\mathbb{C P}^{1}\right)\right)=\pi_{k}(\operatorname{PSL}(2, \mathbb{C}))=\pi_{k}\left(S^{3}\right)$, m.e. $\pi_{2}\left(\mathcal{C}^{n}\left(\mathbb{C P}^{1}\right)\right)=0$ $u \pi_{k}\left(\mathcal{C}^{n}\left(\mathbb{C P}^{1}\right)\right)=\pi_{k}\left(S^{3}\right)$ npu $k>2$.

3.3. Коммутант; эндоморфизмы групп кос. В [24] найдено копредставление коммутанта $B_{n}^{\prime}$ группы $B_{n}$; из него видно, что $B_{n}^{\prime \prime}=B_{n}^{\prime}$ при $n>4$. Так как $B_{n}\left(S^{2}\right)=B_{n} / N$, то $B_{n}^{\prime \prime}\left(S^{2}\right)=B_{n}^{\prime}\left(S^{2}\right)$ при $n>4$. Итак, группь $B_{n}^{\prime}(X)$ совершенны и не имеют нетривиальных гомоморфизмов в свободные группы.

Замечание 1. Равенство $B_{n}^{\prime \prime}=B_{n}^{\prime}$ при $n>4$ легко получить из тождества $\sigma_{3} \sigma_{1}^{-1}=\sigma_{2}^{-1} \sigma_{1}^{-1} \cdot\left[\sigma_{3} \sigma_{1}^{-1}, \sigma_{1} \sigma_{2}^{-1}\right] \cdot \sigma_{1} \sigma_{2}$, найденного Е. А. Гориным в $1990-$ х гг.

Следствие 2. При $n>4$ коммутант $B_{n}^{\prime}(X)$ группы кос $B_{n}(X)$ не имеет нетривиальных гомоморфизмов в группь крашеных кос $P B_{k}(X)$.

Определение 2. Гомоморфизмы групп $\phi, \psi: G \rightarrow H \operatorname{conpяженъ~}(\phi \sim \psi)$, если $\psi(g)=h \phi(g) h^{-1}$ для некоторого $h \in H$ и всех $g \in G$.

Гомоморфизм $\psi: G \rightarrow \mathbf{S}(n)$ транзитивен, если его образ $\psi(G) \subseteq \mathbf{S}(n)$ транзитивно действует на $\{1, \ldots, n\}$. 
Элементы $\sigma_{1}$ и $\alpha=\sigma_{1} \cdots \sigma_{n-1}$ порождают $B_{n}$; то же верно и для $B_{n}\left(S^{2}\right)$.

Артин [10] описал (с точностью до сопряженности) все неабелевы транзитивные гомоморфизмы $\psi: B_{n} \rightarrow \mathbf{S}(n)$ :

Теорема Артина. (а) Ecли $n \neq 4$ u $n \neq 6$, mo $\psi \sim \mu$, где $\mu: B_{n} \rightarrow \mathbf{S}(n)-$ стандартный эпиморбизм, $\mu\left(\sigma_{i}\right)=(i, i+1), 1 \leqslant i \leqslant n-1$.

(b) Ecли $n=6$ и $\psi \nsim \mu$, mо $\psi \sim \nu_{6}$, где $\nu_{6}\left(\sigma_{1}\right)=(1,2)(3,4)(5,6)$ u $\nu_{6}(\alpha)=$ $(1,2,3)(4,5)$.

(с) Если $n=4$ и $\psi \nsim \mu$, то $\psi$ сопряэсен с одним из следующих трех гомоморфизмов $\nu_{4,1}, \nu_{4,2}, \nu_{4,3}: \nu_{4,1}\left(\sigma_{1}\right)=(1,2,3,4), \nu_{4,1}(\alpha)=(1,2) ; \nu_{4,2}\left(\sigma_{1}\right)=$ $(1,3,2,4), \nu_{4,2}(\alpha)=(1,2,3,4) ; \nu_{4,3}\left(\sigma_{1}\right)=(1,2,3), \nu_{4,3}(\alpha)=(1,2)(3,4)$. Если $\psi \sim \nu_{4,3}$, mo $\psi\left(B_{4}\right)=\mathbf{A}(4)$.

(d) Все неабелевы транзитивные гомоморфизмы $\psi: B_{n} \rightarrow \mathbf{S}(n)$, кроме сопряженных с $\nu_{4,3}$, сюргективены.

Лемма Артина. Если $n, m \in \mathbb{N}, n>4$, и существует простое $p>2$, maкое, что $m / 2<p \leqslant n-2$, то для каждого неабелева транзитивного гомоморфизма $\psi: B_{n} \rightarrow \mathbf{S}(m)$ перестановка $\psi\left(\sigma_{1}\right)$ имеет хотя бы $n-2$ неподвиюнных точек.

Вот полезное дополнение к теореме Артина о гомоморфизмах $B_{n} \rightarrow \mathbf{S}(n)$.

Теорема 8. Если $n \neq 4$, то при $k<n$ все гомоморфизмы $B_{n}(X) \rightarrow \mathbf{S}(k)$ абелевы, а каждый неабелев гомоморфизм $B_{n}(X) \rightarrow \mathbf{S}(n)$ сюрвективен.

Доказательство. Так как $B_{n}\left(\mathbb{C P}^{1}\right) \cong B_{n} / S$, достаточно рассмотреть случай $X=\mathbb{C}, B_{n}(X)=B_{n}$. Для $n \leqslant 3$ первое утверждение тривиально. Допустим, что $n>\max \{4, k\}$ и образ $H=\operatorname{Im} \psi$ гомоморфизма $\psi: B_{n} \rightarrow \mathbf{S}(k)$ неабелев. Для каждой $H$-орбиты $O \subseteq\{1, \ldots, k\}$ определим редукиию $\psi_{O}: B_{n} \rightarrow \mathbf{S}(O)$, где $\psi_{O}(g)$ - сужение $\psi(g)$ на $O$. Все редукции транзитивны и хотя бы одна из них, скажем $\psi_{O_{\circ}}: B_{n} \rightarrow \mathbf{S}\left(O_{\circ}\right) \cong \mathbf{S}(m)\left(m=\# O_{\circ}\right)$, неабелева. Так как $n>4$ и $m \leqslant k<n$, то найдется такое простое $p>2$, что $m / 2<p \leqslant n-2$ (теорема Чебышева). По лемме Артина нетривиальная перестановка $\psi_{O_{\circ}}\left(\sigma_{1}\right) \in \mathbf{S}(m)$ имеет хотя бы $n-2$ неподвижных точек, что невозможно, ибо $n-2 \geqslant m-1$.

Рассмотрим теперь неабелев гомоморфизм $\psi: B_{n}(X) \rightarrow \mathbf{S}(n)$. Можно считать, что $\psi$ интранзитивен (иначе его сюръективность следует из теоремы Артина). Стало быть, \#O<n для любой $(\operatorname{Im} \psi)$-орбиты $O \subset\{1, \ldots, n\}$, и из первого утверждения теоремы следует, что редукция гомоморфизма $\psi$ на любую такую орбиту абелева; но тогда $\psi$ абелев, что противоречит предположению.

Замечание 2. При $6<n<k<2 n$ все транзитивные гомоморфизмы $B_{n}(X) \rightarrow \mathbf{S}(k)$ абелевы. C точностью до сопряженности, есть четыре неабелевых транзитивных гомоморфизма из $B_{n}$ в $\mathbf{S}(2 n)$ и два из $B_{n}\left(S^{2}\right)$ в $\mathbf{S}(2 n)$ (в [46], [49] это доказано при $n>8$; случаи $n=7,8$ разобрал С. Ю. Оревков).

Лемма 2. Пусть $n>4$, и пусть $X, Y \in\left\{\mathbb{C}, \mathbb{C P}^{1}\right\}$. Тогда композииия $\psi=$ $\mu \circ \varphi: B_{n}(X) \stackrel{\varphi}{\longrightarrow} B_{k}(Y) \stackrel{\mu}{\longrightarrow} \mathbf{S}(k)$ неабелева гомоморбизма $\varphi: B_{n}(X) \rightarrow B_{k}(Y)$ со стандартным эпиморфизмом $\mu: B_{k}(Y) \rightarrow \mathbf{S}(k)$ является неабелевым гомоморфизмом.

Доказательство. Если $\psi=\mu \circ \varphi$ абелев, то $\mu\left(\varphi\left(B_{n}^{\prime}(X)\right)\right)=\{1\}$ и $\varphi\left(B_{n}^{\prime}(X)\right) \subseteq$ Ker $\mu=P B_{k}(Y)$. Группа $B_{n}^{\prime}(X)$ совершенна, так что из последнего включения и следствия 2 вытекает, что $\varphi\left(B_{n}^{\prime}(X)\right)=\{1\}$ и $\varphi$ абелев, вопреки условию.

Лемма 3. Если $n>4 u \psi: B_{n}(X) \rightarrow \mathbf{S}(n)$ неабелев, то $\operatorname{Ker} \psi=P B_{n}(X)$. 
Доказательство. По теореме 8 гомоморфизм $\psi$ сюръективен; поэтому для $X=\mathbb{C}$ утверждение леммы следует из теоремы Артина. Если же $X=\mathbb{C P}^{1} \cong$ $S^{2}$ и $\theta: B_{n} \rightarrow B_{n}\left(S^{2}\right)$ - естественный эпиморфизм, то, применяя лемму $2 \mathrm{~K}$ композиции $\widetilde{\psi}=\psi \circ \theta: B_{n} \stackrel{\theta}{\longrightarrow} B_{n}\left(S^{2}\right) \stackrel{\psi}{\longrightarrow} \mathbf{S}(n)$, заключаем, что $\theta^{-1}(\operatorname{Ker} \psi)=$ $\operatorname{Ker}(\psi \circ \theta)=\operatorname{Ker} \widetilde{\psi}=P B_{n}$ и $\operatorname{Ker} \psi=\theta\left(\theta^{-1}(\operatorname{Ker} \psi)\right)=\theta\left(P B_{n}\right)=P B_{n}\left(S^{2}\right)$.

Теорема 9. При $n \neq 4 u n>k$ все гомоморфизмы $\varphi: B_{n}(X) \rightarrow B_{k}(X)$ абелевы, а потому абелевы и все непрерывные отображения $\mathcal{C}^{n}(X) \rightarrow \mathcal{C}^{k}(X)$.

Доказательство. Для $n=3$ все ясно. Если $n>4$ и $\varphi$ неабелев, то по лемме 2 неабелев и $\psi=\mu \circ \varphi: B_{n}(X) \stackrel{\varphi}{\longrightarrow} B_{k}(X) \stackrel{\mu}{\longrightarrow} \mathbf{S}(k)$, что противоречит теореме 8 .

3.4. Неабелевы эндоморфизмы группы $B_{n}(X)$ сохраняют $P B_{n}(X)$. Следующая теорема распространяет теорему Артина [10] о сохранении подгруппы $P B_{n}$ автоморфизмами группы $B_{n}$ на неабелевы эндоморфизмы групп $B_{n}$ и $B_{n}\left(S^{2}\right)$.

Теорема 10. Если $n>4$, mо $\varphi^{-1}\left(P B_{n}(X)\right)=P B_{n}(X)$ u, следовательно, $\varphi\left(P B_{n}(X)\right) \subseteq P B_{n}(X)$ для каждого неабелева эндоморбизма $\varphi$ группы $B_{n}(X)$.

Доказательство. По леммам 2 и $3 \psi=\mu \circ \varphi: B_{n}(X) \stackrel{\varphi}{\longrightarrow} B_{n}(X) \stackrel{\mu}{\longrightarrow} \mathbf{S}(n)$ - неабелев гомоморфизм и $P B_{n}(X)=\operatorname{Ker} \psi=\varphi^{-1}(\operatorname{Ker} \mu)=\varphi^{-1}\left(P B_{n}(X)\right)$.

Замечание 3. Вот более сильный результат: если $n>4$, mo $B_{n}^{\prime} \cap P B_{n}$ вполне характеристическая подгруппа группы $B_{n}^{\prime}$; см. [48], [49].

\section{§4. Эндоморфизмы пространств $\mathcal{C}^{n}(X)$ : доказательство теоремы 4 для $X=\mathbb{C}$ или $\mathbb{C P}^{1}$}

\section{1. От эндоморфизмов $\mathcal{C}^{n}(X)$ к эндоморфизмам $\mathcal{C}_{o}^{n}(X)$.}

Определение 3. Отображение $f: \mathcal{C}_{o}^{n}(X) \rightarrow \mathcal{C}_{o}^{n}(X)$ эквивариантно, если найдется $\alpha \in \operatorname{Aut} \mathbf{S}(n)$, такой, что $f(\sigma q)=\alpha(\sigma) f(q)$ при $q \in \mathcal{C}_{o}^{n}(X)$ и $\sigma \in \mathbf{S}(n)$.

Теорема 11. Если $n>4$, то любое неабелево непрерьвное отображение $F: \mathcal{C}^{n}(X) \rightarrow \mathcal{C}^{n}(X)$ включается в коммутативную диаграмму

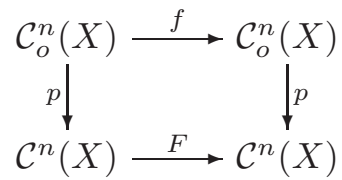

с непрерывным $f$. Если $F$ голоморфно, то $f$ голоморфно и эквивариантно.

Доказательство. Диаграмма (10) (c $f$, голоморфным вместе с $F$ ) существует ввиду теоремы 10. Так как $p$ - накрытие Галуа, то найдется единственный с точностью до сопряженности эндоморфизм $\alpha: \mathbf{S}(n) \rightarrow \mathbf{S}(n)$, такой, что

$$
f(\sigma q)=\alpha(\sigma) f(q) \text { для всех } q \in \mathcal{C}_{o}^{n}(X) \text { и } \sigma \in \mathbf{S}(n) ;
$$

он может быть включен в коммутативную диаграмму с точными строками

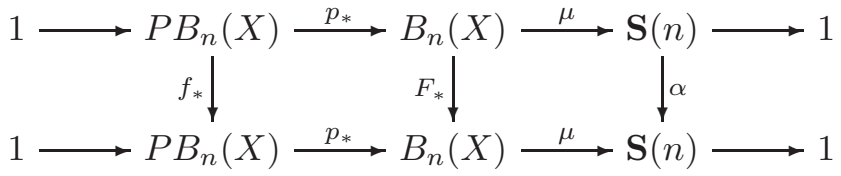


Гомоморфизм $F_{*}$ неабелев, и по лемме 2 неабелевы $\alpha \circ \mu=\mu \circ F_{*}$ и $\alpha$. Так как $n>4$ и $\mathbf{A}(n)$ не может быть ядром неабелева эндоморфизма группы $\mathbf{S}(n)$, то $\operatorname{Ker} \alpha=\{1\}$ и $\alpha$ - автоморфизм. (При $n \neq 6$ все автоморфизмы группы $\mathbf{S}(n)$ внутренние; при $n=6$ «наш» $\alpha$ тоже внутренний; см. замечание 6 в п. 4.7.)

4.2. Эквивариантные эндоморфизмы пространства $\mathcal{C}_{o}^{n}(X):$ план действий. Чтобы доказать, что $F$ ручной, достаточно проверить, что $f$ является ручным в следующем смысле: существуют $\sigma \in \mathbf{S}(n)$ u $\mathbf{S}(n)$-инвариантный морфизм $T_{o}: \mathcal{C}_{o}^{n}(X) \rightarrow$ Aut $X$, такие, что морфизм $\widetilde{f} \stackrel{\text { def }}{=} \sigma^{-1} \circ f$ удовлетворяет условию

$$
\widetilde{f}(q)=T_{o}(q) q \quad \text { для всех } q \in \mathcal{C}_{o}^{n}(X) .
$$

В частности, после подходящей перенумерации координат, для каждой точки $q=\left(q_{1}, \ldots, q_{n}\right) \in \mathcal{C}_{o}^{n}(X)$ и каждого упорядоченного набора попарно различных $i_{1}, \ldots, i_{t(X)+1} \leqslant n$ упорядоченные $(t(X)+1)$-точечные подмножества $\left(f_{i_{1}}(q), \ldots, f_{i_{t(X)+1}}(q)\right) \subset X u\left(q_{i_{1}}, \ldots, q_{i_{t(X)+1}}\right) \subset X$ имеют одинаковый базисный (Aut $X$ )-инвариант $\mathcal{R}_{X}$. Как известно, если $X=\mathbb{C P}^{1}$, то $\mathcal{R}_{X}$ - двойное отношение

$$
\mathcal{R}_{\mathbb{C P}^{1}}(a, b, c, d)=c r(a, b, c, d) \stackrel{\text { def }}{=} \frac{d-a}{d-b}: \frac{a-c}{b-c}, \quad a, b, c, d \in \mathbb{C P}^{1} ;
$$

если же $X=\mathbb{C}$, то $\mathcal{R}_{X}-$ простое отношение

$$
\mathcal{R}_{\mathbb{C}}(a, b, c)=\operatorname{sr}(a, b, c) \stackrel{\text { def }}{=}(a-c) /(b-c)=c r(b, a, c, \infty), \quad a, b, c \in \mathbb{C} .
$$

Итак, для ручного $f$ после подходящей перенумерации координат выполняются следующие условия когерентности:

$$
\begin{aligned}
& \frac{f_{i}(q)-f_{1}(q)}{f_{2}(q)-f_{1}(q)}= \frac{q_{i}-q_{1}}{q_{2}-q_{1}} \\
& \text { для всех } q=\left(q_{1}, \ldots, q_{n}\right) \in \mathcal{C}_{o}^{n}(\mathbb{C}) \text { и всех } i=3, \ldots, n, \\
& \frac{f_{i}(q)-f_{1}(q)}{f_{i}(q)-f_{2}(q)}: \frac{f_{1}(q)-f_{3}(q)}{f_{2}(q)-f_{3}(q)}=\frac{q_{i}-q_{1}}{q_{i}-q_{2}}: \frac{q_{1}-q_{3}}{q_{2}-q_{3}} \\
& \text { для всех } q=\left(q_{1}, \ldots, q_{n}\right) \in \mathcal{C}_{o}^{n}\left(\mathbb{C P}^{1}\right) \text { и всех } i=4, \ldots, n .
\end{aligned}
$$

Мы заметно приблизимся к цели, доказав, что координатные функции всякого эквивариантного эндоморфизма (после подходящей перенумерации) удовлетворяют соотношениям (16) или (17). Доказательство основано на том наблюдении, что простые и двойные отношения координат - единственные непостоянные голоморфные функции $\mathcal{C}_{o}^{n}(X) \rightarrow \mathbb{C} \backslash\{0,1\}$ (при $X=\mathbb{C P}^{1}$ остаются лишь двойные отношения). Аналогичные комбинации координатных функций $f_{i}$ эквивариантного эндоморфизма $f$ пространства $\mathcal{C}_{o}^{n}(X)$ тоже непостоянны и голоморфно отображают $\mathcal{C}_{o}^{n}(X)$ в $\mathbb{C} \backslash\{0,1\}$; значит, и они являются простыми или двойными отношениями коорднат. Исходная аналитическая задача приобретает комбинаторный характер: разобраться, «кто есть кто», т. е. какому именно из простых или двойных отношений координат равно каждое данное простое (двойное) отношение координатных функций эквивариантного эндоморфизма. Этому поможет новый гомоморфно-комбинаторный инвариант комплексных пространств, представляющий также самостоятельный интерес. 
4.3. Функции без двух значений. Следующую лемму можно рассматривать как мультипликативный аналог «abc-леммы» для полиномов от одной переменной. Запись $f \approx g$ означает, что $f=c g$ для некоторого $c \in \mathbb{C}^{*}$.

Лемма 4. Пусть полиномы $P, Q, R \in \mathbb{C}\left[z_{1}, \ldots, z_{n}\right]$ попарно взаимно простъ, не имеют нулей вне большой диагонали $\mathcal{D}=\bigcup_{i \neq j}\left\{z_{i}=z_{j}\right\}$ и хотя бъ один из них непостоянен. Если

$$
P+Q+R=0
$$

mо либо

$$
P \approx z_{q}-z_{r}, \quad Q \approx z_{r}-z_{p}, \quad R \approx z_{p}-z_{q},
$$

либо

$$
P \approx\left(z_{q}-z_{r}\right)\left(z_{s}-z_{p}\right), \quad Q \approx\left(z_{r}-z_{p}\right)\left(z_{s}-z_{q}\right), \quad R \approx\left(z_{p}-z_{q}\right)\left(z_{s}-z_{r}\right) .
$$

Доказательство. В силу $(*)$ хотя бы две из степеней $a_{i}, b_{i}, c_{i}$ полиномов $P, Q, R$ по $z_{i}$ совпадают с $\max \left\{a_{i}, b_{i}, c_{i}\right\}$. Так как $P, Q, R$ не имеют нулей вне

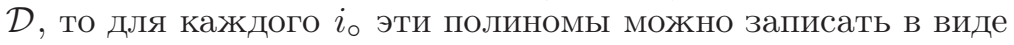

$$
P_{i_{\circ}} \prod_{j \neq i_{\circ}}\left(z_{i_{\circ}}-z_{j}\right)^{a_{i_{\circ} j}}, \quad Q_{i_{\circ}} \prod_{j \neq i_{\circ}}\left(z_{i_{\circ}}-z_{j}\right)^{b_{i_{\circ} j}} \quad \text { и } \quad R_{i_{\circ}} \prod_{j \neq i_{\circ}}\left(z_{i_{\circ}}-z_{j}\right)^{c_{i_{\circ} j}}
$$

соответственно, где полиномы

$$
P_{i_{\circ}} \approx \prod_{\substack{i \neq j \\ i, j \neq i_{\circ}}}\left(z_{i}-z_{j}\right)^{a_{i_{\circ} ; i, j}}, \quad Q_{i_{\circ}} \approx \prod_{\substack{i \neq j \\ i, j \neq i_{\circ}}}\left(z_{i}-z_{j}\right)^{b_{i_{\circ} ; i, j}}, \quad R_{i_{\circ}} \approx \prod_{\substack{i \neq j \\ i, j \neq i_{\circ}}}\left(z_{i}-z_{j}\right)^{c_{i_{\circ} ; i, j}}
$$

попарно взаимно просты и не содержат $z_{i_{\circ}}$, а произведения $\prod_{j \neq i_{\circ}}\left(z_{i_{\circ}}-z_{j}\right)^{a_{i_{\circ} j}}$, $\prod_{j \neq i_{\circ}}\left(z_{i_{\circ}}-z_{j}\right)^{b_{i_{\circ} j}}$ и $\prod_{j \neq i_{\circ}}\left(z_{i_{\circ}}-z_{j}\right)^{c_{i_{\circ} j}}$ - попарно взаимно простые полиномы по $z_{i_{\circ}}$ степеней $a_{i_{\circ}}, b_{i_{\circ}}, c_{i_{\circ}}$. Есть лишь две возможности: (а) для некоторого $i=p$ хотя бы два из чисел $a_{p}, b_{p}, c_{p}$ различны и $(\mathrm{b}) a_{i}=b_{i}=c_{i}$ для всех $i$.

В случае (а) можно считать, что $a_{p}<b_{p}=c_{p}$. Учитывая $(20)\left(\right.$ с $\left.i_{\circ}=p\right)$ и принимая в расчет лишь старшие по $z_{p}$ члены в $(*)$, получим $Q_{p}+R_{p}=0$; так как $Q_{p}$ и $R_{p}$ взаимно просты, то они - ненулевые постоянные. Поэтому $Q \approx \prod_{j \neq p}\left(z_{p}-z_{j}\right)^{b_{p j}}, R \approx \prod_{j \neq p}\left(z_{p}-z_{j}\right)^{c_{p j}}$ и $(*)$ превращается в

$$
P_{p} \prod_{j \neq p}\left(z_{p}-z_{j}\right)^{a_{p j}}+B \prod_{j \neq p}\left(z_{p}-z_{j}\right)^{b_{p j}}+C \prod_{j \neq p}\left(z_{p}-z_{j}\right)^{c_{p j}}=0,
$$

где $B, C \in \mathbb{C}^{*}$. Все $a_{p j}$ равны 0 (если некое $a_{p j}$ положительно, то $z_{p}-z_{j}$ не входит в два других слагаемых и (22) невозможно). Значит, $a_{p}=\sum_{j \neq p} a_{p j}=0$, $P$ не содержит $z_{p}, P=P_{p}$ и $(22)$ принимает вид $P+B \prod_{j \neq p}\left(z_{p}-z_{j}\right)^{b_{p j}}+$ $C \prod_{j \neq p}\left(z_{p}-z_{j}\right)^{c_{p j}}=0$. При $z_{p}=0$ это дает $P+B \prod_{j \neq p}\left(-z_{j}\right)^{b_{p j}}+C \prod_{j \neq p}\left(-z_{j}\right)^{c_{p j}}$ $=0$, где полином $P=P_{p}$ определен в $(21)\left(\right.$ с $\left.i_{\circ}=p\right)$, a $\prod_{j \neq p}\left(-z_{j}\right)^{b_{p j}}$ и $\prod_{j \neq p}\left(-z_{j}\right)^{c_{p j}}$ взаимно просты. Такое возможно, лишь если $P, Q, R$ удовлетворяют (18) с попарно различными $p, q, r$.

В случае (b) есть $z_{s}$, входящее в $P, Q$ и $R$. Учитывая $(20)$ и $(21)\left(\right.$ с $\left.i_{\circ}=s\right)$, представим $(*)$ в виде

$$
P_{s} \prod_{j \neq s}\left(z_{s}-z_{j}\right)^{a_{s j}}+Q_{s} \prod_{j \neq s}\left(z_{s}-z_{j}\right)^{b_{s j}}+R_{s} \prod_{j \neq s}\left(z_{s}-z_{j}\right)^{c_{s j}}=0,
$$


где $P_{s}, Q_{s}, R_{s}$ не содержат $z_{s}$ и $\sum_{j \neq s} a_{s j}=\sum_{j \neq s} b_{s j}=\sum_{j \neq s} c_{s j}>0$; значит, $a_{s p}>0$ для некоторого $p \neq s$. Так как $P$ взаимно прост с $Q$ и $R$ и содержит $\left(z_{s}-z_{p}\right)^{a_{s p}}$, то $b_{s p}=c_{s p}=0$. Беря в расчет в $(23)$ лишь старшие по $z_{s}$ члены, получим $P_{s}+Q_{s}+R_{s}=0$; покажем, что $P_{s}, Q_{s}, R_{s}$ - тройка типа (а). Пусть $a_{s p}^{\prime}, b_{s p}^{\prime}, c_{s p}^{\prime}$ - степени полиномов $P_{s}, Q_{s}$ и $R_{s}$ по выбранному выше $z_{p}$; тогда $a_{s p}^{\prime}=a_{p}-a_{s p}<a_{p}, b_{s p}^{\prime}=b_{p}-b_{s p}=b_{p}=a_{p}$ и $c_{s p}^{\prime}=c_{p}-c_{s p}=c_{p}=a_{p}$. Стало быть, $a_{s p}^{\prime}<b_{s p}^{\prime}=c_{s p}^{\prime}$ и $Q_{s}, R_{s} \neq$ const, что нам и требовалось. Итак, $P_{s} \approx\left(z_{q}-z_{r}\right), Q_{s} \approx\left(z_{r}-z_{p}\right)$ и $R_{s} \approx\left(z_{p}-z_{q}\right)$ для двух различных $q, r$, таких, что $q \neq p, s$ и $r \neq p, s$, и (23) можно записать как

$$
\begin{aligned}
& A\left(z_{q}-z_{r}\right)\left(z_{s}-z_{p}\right)^{a_{s p}} \prod_{j \neq s, p}\left(z_{s}-z_{j}\right)^{a_{s j}} \\
& \quad+B\left(z_{r}-z_{p}\right) \prod_{j \neq s, p}\left(z_{s}-z_{j}\right)^{b_{s j}}+C\left(z_{p}-z_{q}\right) \prod_{j \neq s, p}\left(z_{s}-z_{j}\right)^{c_{s j}}=0
\end{aligned}
$$

с некоторыми $A, B, C \in \mathbb{C}^{*}$. В частности, на подпространстве $z_{p}=z_{q}$ имеем $A\left(z_{q}-z_{r}\right)\left(z_{s}-z_{q}\right)^{a_{s p}} \prod_{j \neq s, p}\left(z_{s}-z_{j}\right)^{a_{s j}}+B\left(z_{r}-z_{q}\right) \prod_{j \neq s, p}\left(z_{s}-z_{j}\right)^{b_{s j}}=0$, так что $A\left(z_{s}-z_{q}\right)^{a_{s p}} \prod_{j \neq s, p}\left(z_{s}-z_{j}\right)^{a_{s j}}-B \prod_{j \neq s, p}\left(z_{s}-z_{j}\right)^{b_{s j}}=0$. Произведения $\prod_{j \neq s, p}\left(z_{s}-z_{j}\right)^{a_{s j}}$ и $\prod_{j \neq s, p}\left(z_{s}-z_{j}\right)^{b_{s j}}$ должны быть взаимно просты; значит, $A=B, a_{s j}=0$ при $j \neq s, p, b_{s j}=0$ при $j \neq s, p, q$ и $a_{s p}=b_{s q}$. Поэтому $P \approx\left(z_{q}-z_{r}\right)\left(z_{s}-z_{p}\right)^{a_{s p}}, Q \approx\left(z_{r}-z_{p}\right)\left(z_{s}-z_{q}\right)^{a_{s p}}, R \approx\left(z_{p}-z_{q}\right) \prod_{j \neq s, p, q}\left(z_{s}-z_{j}\right)^{c_{s j}}$ и (24) принимает вид $A\left(z_{q}-z_{r}\right)\left(z_{s}-z_{p}\right)^{a_{s p}}+A\left(z_{r}-z_{p}\right)\left(z_{s}-z_{q}\right)^{a_{s p}}+C R=0$. Ввиду $a_{q}=b_{q}=c_{q}$ и $a_{r}=b_{r}=c_{r}$ получаем $a_{s p}=1, c_{s j}=0$ для всех $j \neq r$ и $c_{s r}=1$; итак, $P \approx\left(z_{q}-z_{r}\right)\left(z_{s}-z_{p}\right), Q \approx\left(z_{r}-z_{p}\right)\left(z_{s}-z_{q}\right), R \approx\left(z_{p}-z_{q}\right)\left(z_{s}-z_{r}\right)$.

Теорема 12. Непостоянная голоморфная функиия $\mu: \mathcal{C}_{o}^{n}(\mathbb{C}) \rightarrow \mathbb{C} \backslash\{0,1\}$ является простым или двойным отношением координат. Все непостоянные голоморфные функиии $\mathcal{C}_{o}^{n}\left(\mathbb{C P}^{1}\right) \rightarrow \mathbb{C} \backslash\{0,1\}$ - двойные отношения координат.

Доказательство. По теореме Пикара такая функция $\mu$ рациональна на каждой комплексной прямой $\ell \subset \mathbb{C}^{n}$, не содержащейся в $\bigcup_{i \neq j}\left\{z_{i}=z_{j}\right\}$. Значит, $\mu$ рациональна на $\mathbb{C}^{n}$ и регулярна на $\mathcal{C}_{o}^{n}(\mathbb{C})$, т. е. $\mu=-P / R$, где $P, R$ - взаимно простые полиномы без нулей в $\mathcal{C}_{o}^{n}(\mathbb{C})$ и хоть один из них непостоянен. Полином $Q=-P-R$ взаимно прост с $P$ и $R$; как и $-Q / R=1-\mu$, он не имеет нулей в $\mathcal{C}_{o}^{n}(\mathbb{C})$. Итак, к $P, Q$ и $R$ применима лемма 4 , так что $\mu=-P / R-$ простое или двойное отношение координат. $\mathrm{Ha}_{o}^{n}\left(\mathbb{C P}^{1}\right)$ «выживают» лишь двойные отношения, ибо $\left(q_{k}-q_{i}\right) /\left(q_{k}-q_{j}\right)$ имеет полюс вдоль дивизора $q_{i}=\infty$.

Следствие 3. Пусть $X=\mathbb{C}$ или $\mathbb{C P}^{1}$. Тогда все непостоянные простые $и$ двойные отношения координатных функиий морфизма $\mathcal{C}_{o}^{n}(X) \rightarrow \mathcal{C}_{o}^{k}(\mathbb{C})$ и все непостоянные двойные отношения координатных бункиий морфизма $\mathcal{C}_{o}^{n}(X) \rightarrow$ $\mathcal{C}_{o}^{k}\left(\mathbb{C P}^{1}\right)$ являются простыми или двойными отношениями координат в $\mathcal{C}_{o}^{n}(X)$.

Замечание 4. Каждая голоморфная функция $\operatorname{PSL}(2, \mathbb{C}) \times \mathcal{C}_{o}^{n-3}(\mathbb{C} \backslash\{0,1\}) \cong$ $\mathcal{C}_{o}^{n}\left(\mathbb{C P}^{1}\right) \rightarrow \mathbb{C} \backslash\{0,1\}$ постоянна на всех $\mathbf{P S L}(2, \mathbb{C})$-слоях. Поэтому теорема 12 приводит также к следующему описанию всех непостоянных голоморфных 
функиий $h: \mathcal{C}_{o}^{n-3}(\mathbb{C} \backslash\{0,1\}) \rightarrow \mathbb{C} \backslash\{0,1\}:$

$$
h\left(q_{1}, \ldots, q_{n-3}\right)=h_{i, j, k, l}\left(q_{1}, \ldots, q_{n}\right)=\frac{q_{i}-q_{j}}{q_{i}-q_{k}}: \frac{q_{j}-q_{l}}{q_{k}-q_{l}},
$$

где $i, j, k, l \leqslant n$ попарно различны, $q_{n-2}=0, q_{n-1}=1$ u $q_{n}=\infty$. Сходным приемом можно описать все голоморфные функции $\left.\mathcal{C}_{o}^{n}\left(\mathbb{C}^{*}\right) \rightarrow \mathbb{C} \backslash\{0,1\} .{ }^{1}\right) \triangle$

4.4. Комплекс $\mathscr{C}(\boldsymbol{Z}, \boldsymbol{G})$. Пусть $\operatorname{Hol}(Z, G)$ - группа всех голоморфных отображений комплексного пространства $Z$ в связную комплексную группу Ли $G$ (умножение поточечно), $\mathcal{V}(Z, G)=\{\lambda \in \operatorname{Hol}(Z, G) \mid \lambda \neq \operatorname{const}, \lambda(Z) \not \supset 1\}$, $\mathcal{E}(Z, G)=\left\{\{\mu, \nu\} \subseteq \mathcal{V}(Z, G) \mid \mu \cdot \nu^{-1} \in \mathcal{V}(Z, G)\right\}, \mathcal{G}(Z, G)$ - граф с множеством вершин $\mathcal{V}(Z, G)$ и множеством ребер $\mathcal{E}(Z, G)$ и, наконец, $\mathscr{C}(Z, G)-$ флаг-комплекс графа $\mathcal{G}(Z, G)$ (его симплексы - все конечные подмножества $\Delta \subseteq \mathcal{V}(Z, G)$, в которых любая пара различных вершин $\mu, \mu^{\prime} \in \Delta$ соединена в $\mathcal{G}(\bar{Z}, G)$ ребром). Если $\{\mu, \nu\} \in \mathcal{E}(Z, G)$, то $\mu$ и $\nu$ - собственные делители друг друга: $\nu \mid \mu$ и $\mu \mid \nu$. Если фиксировать $G$, то $\mathcal{G}(Z, G)$ и $\mathscr{C}(Z, G)$ инвариантны относительно биголоморфных изоморфизмов $Z \cong Z^{\prime}$.

Лемма 5. Пусть $f: Z \rightarrow Y$ - голоморбное отображение неприводимъх квазипроективных многообразий. Если для каждого $\lambda \in \mathcal{V}(Y, G)$ композииия $f^{*}(\lambda) \stackrel{\text { def }}{=} \lambda \circ f: Z \stackrel{f}{\longrightarrow} Y \stackrel{\lambda}{\longrightarrow} G \backslash\{1\}$ непостоянна (это заведомо так, если $f$ доминантно), то отображсние вершин $f^{*}: \mathcal{V}(Y, G) \ni \lambda \mapsto \lambda \circ f \in \mathcal{V}(Z, G)$ индуиирует симплициальное отображение $f^{*}: \mathscr{C}(Y, G) \rightarrow \mathscr{C}(Z, G)$, которое на каждом симплексе ингективно и потому сохраняет его размерность.

Доказательство. Если $\mu \neq \nu$ - вершины симплекса $\Delta \in \mathscr{C}(Y, G)$, то $\lambda \stackrel{\text { def }}{=}$ $\mu \cdot \nu^{-1} \in \mathcal{V}(Y, G), f^{*}(\mu) \cdot\left(f^{*}(\nu)\right)^{-1}=f^{*}(\lambda) \neq$ const и $\mu(f(z)) \cdot \nu^{-1}(f(z))=$ $\lambda(f(z)) \neq 1$ при $z \in Z$, так что $f^{*}(\nu) \mid f^{*}(\mu)$. Значит, $f^{*}$ инъективно на симплексе $\Delta$ и $f^{*}(\Delta) \in \mathscr{C}(Z, G)$. Последнее утверждение леммы очевидно.

Итак, $Z \mapsto \mathscr{C}(Z, G)$ - контравариантный функтор из категории неприводимых квазипроективных многообразий и доминантных отображений в категорию симплициальных комплексов и симплициальных отображений. Морфизмы $Z \rightarrow G \backslash\{1\}$ можно заменить классами их голоморфной гомотопии. Инварианты комплекса $\mathscr{C}(Z, G)$ являются инвариантами биголоморфных отображений многообразия $Z$.

4.5. Комплекс простых и двойных отношений. В поле $\mathbb{C}(q)$ комплексных рациональных функций от независимых переменных $\mathbf{q}=\left\{q_{i} \mid i \in \mathbb{N}\right\}$ положим

$$
s r_{i j k}=\operatorname{sr}\left(q_{i}, q_{j}, q_{k}\right)=\frac{q_{i}-q_{k}}{q_{j}-q_{k}} \quad \text { и } \quad c r_{i j k l}=c r\left(q_{i}, q_{j}, q_{k}, q_{l}\right)=\frac{q_{l}-q_{i}}{q_{l}-q_{j}}: \frac{q_{i}-q_{k}}{q_{j}-q_{k}} .
$$

Пусть $\mathcal{V}_{s r}(\mathbf{q})$ и $\mathcal{V}_{c r}(\mathbf{q})$ - множества всех $s r$ и $c r$, и пусть $\mathcal{V}(\mathbf{q})=\mathcal{V}_{s r}(\mathbf{q}) \cup \mathcal{V}_{c r}(\mathbf{q})$. Носитель $\operatorname{supp} \mu$ функции $\mu \in \mathcal{V}(\mathbf{q})$ - это множество всех входящих в $\mu$ переменных. Есть 6 простых отношений с носителем $\left\{q_{i}, q_{j}, q_{k}\right\}$ и 6 двойных отношений с носителем $\left\{q_{i}, q_{j}, q_{k}, q_{l}\right\}$. Запись $\nu \mid \mu$ означает, что $\mu, \nu, \mu / \nu \in \mathcal{V}(\mathbf{q})$; тогда $\nu / \mu \in \mathcal{V}(\mathbf{q})$ и $\mu, \nu$ - собственные делители друг друга; $C D\left(\mu, \mu^{\prime}\right)-$ множество всех общих собственных делителей функций $\mu$ и $\mu^{\prime}$. Пусть $\mathcal{G}(\mathbf{q})-$ граф

1)Это сделано в [30], [32] и [64], но без удобного единообразия, доставляемого леммой 4. 
с множеством вершин $\mathcal{V}(\mathbf{q})$ и множеством ребер $\mathcal{E}(\mathbf{q})=\{\{\mu, \nu\} \subseteq \mathcal{V}(\mathbf{q})|\mu| \nu\}$, $\mathscr{C}(\mathbf{q})$ - его флаг-комплекс. Носитель $\operatorname{supp} \Delta$ симплекса $\Delta-$ это $\bigcup_{\mu \in \Delta} \operatorname{supp} \mu$.

Есть четыре типа симплексов: 1) $S R$-чистые: все $\mu_{i}$ и все $\mu_{i} / \mu_{j}$ принадлежат $\mathcal{V}_{s r}(\mathbf{q})$; они образуют подкомплекс $\left.\mathscr{C}_{s r}(\mathbf{q}) ; 2\right) C R$-чистье: все $\mu_{i}$ принадлежат $\mathcal{V}_{c r}(\mathbf{q})$ (тогда и все $\mu_{i} / \mu_{j}$ принадлежат $\left.\mathcal{V}_{c r}(\mathbf{q})\right)$; они образуют подкомплекс $\left.\mathscr{C}_{c r}(\mathbf{q}) ; 3\right)$ смешанные: есть вершины из $\mathcal{V}_{s r}(\mathbf{q})$ и из $\left.\mathcal{V}_{c r}(\mathbf{q}) ; 4\right)$ все остальные: вершины лежат в $\mathcal{V}_{s r}(\mathbf{q})$, а их отношения - в $\mathcal{V}_{c r}(\mathbf{q})$ (скажем, $\Delta^{m}=\left\{s r_{1,2,3}, s r_{1,2,4}, \ldots, s r_{1,2, m+3}\right\}$; они нам не понадобятся).

Пусть $\mathscr{C}\left(\mathbf{q}_{n}\right), \mathscr{C}_{s r}\left(\mathbf{q}_{n}\right)$ и $\mathscr{C}_{c r}\left(\mathbf{q}_{n}\right)$ - подкомплексы в $\mathscr{C}(\mathbf{q}), \mathscr{C}_{s r}(\mathbf{q})$ и $\mathscr{C}_{c r}(\mathbf{q})$, состоящие из всех их симплексов с носителями в $\mathbf{q}_{n} \stackrel{\text { def }}{=}\left\{q_{1}, \ldots, q_{n}\right\}$; аналогично определим множества вершин $\mathcal{V}\left(\mathbf{q}_{n}\right), \mathcal{V}_{s r}\left(\mathbf{q}_{n}\right), \mathcal{V}_{c r}\left(\mathbf{q}_{n}\right)$. Следующие три леммы доказываются непосредственной проверкой.

Лемма 6. (а) Если $\mu, \nu u \mu / \nu \in \mathcal{V}_{s r}(\mathbf{q})$, то либо знаменатели вершин $\mu u$ $\nu$, либо их числители совпадают; в частности, \# $(\operatorname{supp} \mu \cap \operatorname{supp} \nu)=2$.

(b) Все собственные делители $\mu \in \mathcal{V}_{s r}(\mathbf{q})$ функиии $s r_{i j k}$ получаются заменой $i$ или $j$ на $l \neq i, j, k$; с точностью до выбора $l$ и порядка сомножителей, каждое разложение $s r_{i j k}$ на два множителя из $\mathcal{V}_{s r}(\mathbf{q})$ имеет вид $s r_{i j k}=$ $s r_{i l k} \cdot s r_{l j k}$.

(с) Для любых попарно различных $i, j, k, l \in \mathbb{N}$

$$
\begin{aligned}
& C D\left(s r_{i j k}, s r_{i l k}\right) \cap \mathcal{V}_{s r}(\mathbf{q})=\left\{s r_{i m k} \mid m \neq i, j, k, l\right\}, \\
& C D\left(s r_{i j k}, s r_{l j k}\right) \cap \mathcal{V}_{s r}(\mathbf{q})=\left\{s r_{m j k} \mid m \neq i, j, k, l\right\} .
\end{aligned}
$$

(d) Eсли $\Delta=\{\lambda, \mu, \nu\} \subset \mathcal{V}_{s r}(\mathbf{q})$ - симплекс, то либо знаменатели, либо числители вершин $\lambda, \mu, \nu$ совпадают; в любом случае \# $\operatorname{supp} \Delta=5$.

(е) Если $n \geqslant 3$ и $\Delta \in \mathscr{C}_{s r}\left(\mathbf{q}_{n}\right)$, то либо знаменатели, либо числители всех вершин симплекса $\Delta$ совпадают. Наконеи, $\operatorname{dim} \Delta \leqslant n-3 u \operatorname{dim} \mathscr{C}_{s r}\left(\mathbf{q}_{n}\right) \leqslant n-3$.

Лемма 7. (а) Ecли $\mu, \nu \in \mathcal{V}_{c r}$ (q) $u \nu \mid \mu, m o \#(\operatorname{supp} \mu \cap \operatorname{supp} \nu)=3$.

(b) Все собственные делители $\mu \in \mathcal{V}_{c r}(\mathbf{q})$ функиии cr $_{i j k l}$ получаются заменой одного из индексов $i, j, k, l$ некоторым $m \neq i, j, k, l ;$; точностью до выбора m и порядка сомножителей, формуль $\mathrm{cr}_{i j k l}=c r_{i j k m} \cdot c r_{i j m l}=c r_{i m k l} \cdot c r_{m j k l}$ дают все разложения $\mathrm{cr}_{i j k l}$ в произведение двух двойных отношений.

(с) Для любых пяти различных $i, j, k, l, m \in \mathbb{N}$

$$
C D\left(c r_{i j k l}, c r_{i j k m}\right) \cap \mathcal{V}_{c r}(\mathbf{q})=\left\{c r_{i j k t} \mid t \neq i, j, k, l, m\right\}
$$

еще три аналогичных соотношения получаются из (28) клейновыми перестановками $(i, j)(k, l),(i, k)(j, l)$ u $(i, l)(j, k)$ индексов $i, j, k, l$.

(d) Ecли $\Delta=\{\lambda, \mu, \nu\} \subset \mathcal{V}_{c r}(\mathbf{q})-$ cuмnлекс, mо \# $\operatorname{supp} \Delta=6$.

(e) $\operatorname{dim} \mathscr{C}_{c r}\left(\mathbf{q}_{n}\right) \leqslant n-4$ nри всех $n \geqslant 4$.

Лемма 8. Каждый смешанный симплекс $\Delta$ имеет ровно одну вершину $\mu \in$ $\mathcal{V}_{\text {sr }}(\mathbf{q}) ;$ кроме того, $\operatorname{dim} \Delta \leqslant n-3$, коль скоро $\operatorname{supp} \Delta \subseteq\left\{q_{1}, \ldots, q_{n}\right\}$.

Теорема 12 позволяет отождествить $\mathscr{C}\left(\mathcal{C}_{o}^{n}(\mathbb{C})\right) \stackrel{\text { def }}{=} \mathscr{C}\left(\mathcal{C}_{o}^{n}(\mathbb{C}), \mathbb{C}^{*}\right)$ с $\mathscr{C}\left(\mathbf{q}_{n}\right)$, a $\mathscr{C}\left(\mathcal{C}_{o}^{n}\left(\mathbb{C P}^{1}\right)\right) \stackrel{\text { def }}{=} \mathscr{C}\left(\mathcal{C}_{o}^{n}\left(\mathbb{C P}^{1}\right), \mathbb{C}^{*}\right)-\mathrm{c} \mathscr{C}_{c r}\left(\mathbf{q}_{n}\right)$.

Следствие 4. $\operatorname{dim} \mathscr{C}\left(\mathcal{C}_{o}^{n}(\mathbb{C})\right)=\operatorname{dim} \mathscr{C}_{s r}\left(\mathcal{C}_{o}^{n}(\mathbb{C})\right)=\operatorname{dim} \mathscr{C}_{s r}\left(\mathbf{q}_{n}\right)=n-3 u$ $\operatorname{dim} \mathscr{C}\left(\mathcal{C}_{o}^{n}\left(\mathbb{C P}^{1}\right)\right)=\operatorname{dim} \mathscr{C}_{c r}\left(\mathcal{C}_{o}^{n}\left(\mathbb{C P}^{1}\right)\right)=\operatorname{dim} \mathscr{C}_{c r}\left(\mathbf{q}_{n}\right)=n-4$ 
Доказательство. $\operatorname{dim}\left\{s r_{3,2,1}, \ldots, s r_{n, 2,1}\right\}=\operatorname{dim}\left\{s r_{1,2,3}, c r_{1,2,4,3}, \ldots, c r_{1,2, n, 3}\right\}$ $=n-3$ и $\operatorname{dim}\left\{c r_{1,2,3,4}, \ldots, c r_{1,2,3, n}\right\}=n-4$; остается сослаться на леммы 6,7 и 8.

4.6. $\mathbf{S}(\boldsymbol{n})$-действие в $\mathscr{C}\left(\mathbf{q}_{\boldsymbol{n}}\right)$. Переставляя $q_{1}, \ldots, q_{n}$, получим симплициальное действие группы $\mathbf{S}(n)$ в $\mathcal{V}\left(\mathbf{q}_{n}\right)$, транзитивное на $\mathcal{V}_{s r}\left(\mathbf{q}_{n}\right)$ и $\mathcal{V}_{c r}\left(\mathbf{q}_{n}\right)$, сохраняющее тип и размерность симплексов и подкомплексы $\mathscr{C}_{s r}\left(\mathbf{q}_{n}\right)$ и $\mathscr{C}_{c r}\left(\mathbf{q}_{n}\right)$. Пусть $\mathbf{S}(n)$-инвариантные подмножества $\mathscr{C}_{s r}^{m}\left(\mathbf{q}_{n}\right) \subseteq \mathscr{C}_{s r}\left(\mathbf{q}_{n}\right)$ и $\mathscr{C}_{c r}^{m}\left(\mathbf{q}_{n}\right) \subseteq \mathscr{C}_{c r}\left(\mathbf{q}_{n}\right)$ состоят из всех $m$-мерных симплексов соответствующего типа (это не $m$-мерные остовы!).

Назовем нормальными следующие три упорядоченных $m$-симплекса:

$$
\begin{aligned}
\Delta_{s r}^{m} & =\left[s r_{3,2,1}, s r_{4,2,1}, \ldots, s r_{m+3,2,1}\right] \in \mathscr{C}_{s r}\left(\mathbf{q}_{n}\right) & & (0 \leqslant m \leqslant n-3), \\
\Delta_{s r}^{-m} & =\left[s r_{2,3,1}, s r_{2,4,1}, \ldots, s r_{2, m+3,1}\right] \in \mathscr{C}_{s r}\left(\mathbf{q}_{n}\right) & & (0 \leqslant m \leqslant n-3), \\
\Delta_{c r}^{m} & =\left[c r_{1,2,3,4}, c r_{1,2,3,5}, \ldots, c r_{1,2,3, m+4}\right] \in \mathscr{C}_{c r}\left(\mathbf{q}_{n}\right) & & (0 \leqslant m \leqslant n-4) .
\end{aligned}
$$

Лемма 9. (а) Если $m \geqslant 1, m o \mathscr{C}_{s r}^{m}\left(\mathbf{q}_{n}\right)=\mathbf{S}(n) \Delta_{s r}^{m} \cup \mathbf{S}(n) \Delta_{s r}^{-m}$.

(b) Действие группь $\mathbf{S}(n)$ на $\mathscr{C}_{c r}^{m}\left(\mathbf{q}_{n}\right)$ транзитивно.

Доказательство. (а) Пусть $\Delta=\left[\mu_{0}, \ldots, \mu_{m}\right] \in \mathscr{C}_{s r}^{m}\left(\mathbf{q}_{n}\right), \mu_{0}=s r_{3,2,1}$. Coгласно лемме $6(\mathrm{~b}), \mu_{1}=s r_{l, 2,1}$ или $\mu_{1}=s r_{3, l, 1}(l>3)$. Переставляя $4, \ldots, n$, можно сохранить $\mu_{0}$ и перевести $\mu_{1}$ в одну из функций $s r_{4,2,1}, s r_{3,4,1}$. В первом случае $\left[\mu_{0}, \mu_{1}\right]=\left[s r_{3,2,1}, s r_{4,2,1}\right]$; во втором, применяя транспозицию $(2,3)$ ко всем вершинам симплекса $\Delta$, получим $\left[\mu_{0}, \mu_{1}\right]=\left[s r_{2,3,1}, s r_{2,4,1}\right]$. Если $m=1$, то все кончено. При $m \geqslant 2$ либо

(i) $\Delta=\left[\mu_{0}, \mu_{1}, \mu_{2}, \ldots, \mu_{m}\right]=\left[s r_{3,2,1}, s r_{4,2,1}, \mu_{2}, \ldots, \mu_{m}\right]$, либо

(ii) $\Delta=\left[\mu_{0}, \mu_{1}, \mu_{2}, \ldots, \mu_{m}\right]=\left[s r_{2,3,1}, s r_{2,4,1}, \mu_{2}, \ldots, \mu_{m}\right]$.

В случае (i) у $\mu_{0}$ и $\mu_{1}$ общий знаменатель $q_{1}-q_{2}$. По лемме $6\left(\right.$ e),(c) у $\mu_{2}, \ldots, \mu_{m}$ тот же знаменатель, так что $\mu_{2}=s r_{k_{2}, 2,1}, \ldots, \mu_{m}=s r_{k_{m}, 2,1}$ с попарно различными $k_{2}, \ldots, k_{m} \geqslant 5$; подходящая перестановка $\sigma \in \mathbf{S}\{5, \ldots, n\}$ сохраняет $\mu_{0}, \mu_{1}$ и переводит $\Delta$ в $\Delta_{s r}^{m}$. В случае (ii) у $\mu_{0}, \mu_{1}, \ldots, \mu_{m}$ общий числитель $q_{1}-q_{2}$; как выше, $\mu_{2}=s r_{2, k_{2}, 1}, \ldots, \mu_{m}=s r_{2, k_{m}, 1}$ и $\sigma \Delta=\Delta_{s r}^{-m}$ для подходящего $\sigma \in \mathbf{S}\{5, \ldots, n\}$.

(b) Пусть $\Delta=\left[\mu_{0}, \mu_{1}, \ldots\right] \in \mathscr{C}_{c r}^{m}\left(\mathbf{q}_{n}\right), \mu_{0}=c r_{1,2,3,4}$. По лемме 7(b) $\mu_{1}$ одна из функций $c r_{1,2,3, l}, c r_{1,2, l, 4}, c r_{1, l, 3,4}, c r_{l, 2,3,4}$ c $l \geqslant 5$. Некоторая перестановка $\sigma \in \mathbf{S}\{5, \ldots, n\}$ сохраняет $\mu_{0}$ и переводит $\mu_{1}$ в одну из функций $c r_{1,2,3,5}, c r_{1,2,5,4}, c r_{1,5,3,4}, c r_{5,2,3,4}$. Перестановка $(1,3)(2,4)$ сохраняет $c r_{1,2,3,4}$ и переводит $c r_{1,2,3,5}$ в $c r_{1,5,3,4}$ и $c r_{1,2,5,4}$ в $c r_{5,2,3,4}$; остаются лишь возможности $\left[\mu_{0}, \mu_{1}\right]=\left[c r_{1,2,3,4}, c r_{1,2,3,5}\right]$ или $\left[c r_{1,2,3,4}, c r_{1,2,5,4}\right]$, меняющиеся местами при перестановке $(1,2)(3,4)$. Итак, можно считать, что $\Delta=\left[c r_{1,2,3,4}, c r_{1,2,3,5}, \ldots\right]$. Если $m=1$, то все кончено. При $m \geqslant 2$ соотношение (28) показывает, что $\mu_{2}, \ldots, \mu_{m}$ - это $c r_{1,2,3, k_{2}}, \ldots, c r_{1,2,3, k_{m}}$ с попарно различными $k_{2}, \ldots, k_{m} \geqslant 6$. Подходящая перестановка $\sigma \in \mathbf{S}\{6, \ldots, n\}$ сохраняет $\mu_{0}, \mu_{1}$ и переводит $\Delta$ в $\Delta_{c r}^{m}$.

Лемма 10. Пусть $f: \mathcal{C}_{o}^{n}(X) \rightarrow \mathcal{C}_{o}^{n}(X)$ - эквивариантный эндоморфизм. Тогда для любой непостоянной голоморфной функиии $\mu: \mathcal{C}_{o}^{n}(X) \rightarrow \mathbb{C} \backslash\{0,1\}$ композиция $f^{*}(\mu) \stackrel{\text { def }}{=} \mu \circ f: \mathcal{C}_{o}^{n}(X) \stackrel{f}{\longrightarrow} \mathcal{C}_{o}^{n}(X) \stackrel{\mu}{\longrightarrow} \mathbb{C} \backslash\{0,1\}$ непостоянна.

Доказательство. Если $\mu \circ f=c=$ const $\neq 0,1$, то $(\mu \circ f)(\sigma q) \equiv c$ для всех $\sigma \in \mathbf{S}(n)$. Так как $f$ эквивариантен, найдется $\alpha \in \operatorname{Aut} \mathbf{S}(n)$, такой, что $f(\gamma q)=$ 
$\alpha(\gamma) f(q)$ для всех $\gamma \in \mathbf{S}(n)$ и $q \in \mathcal{C}_{o}^{n}(X)$, так что $c \equiv \mu(f(\gamma q))=\mu(\alpha(\gamma) f(q))$. По теореме 12 вершина $\mu$ принадлежит одному из множеств $\mathcal{V}_{s r}\left(\mathbf{q}_{n}\right), \mathcal{V}_{c r}\left(\mathbf{q}_{n}\right)$, инвариантных относительно инволюций $\lambda \mapsto \lambda^{-1}$ и $\lambda \mapsto 1-\lambda$. Действие группы $\mathbf{S}(n)$ транзитивно на каждом из них; поэтому $\mu^{-1}=s \mu$ и $1-\mu=t \mu$ для некоторых $s, t \in \mathbf{S}(n)$. Выбирая $\sigma=\alpha^{-1}\left(s^{-1}\right)$ и $\tau=\alpha^{-1}\left(t^{-1}\right)$, приходим к несовместимым равенствам $c^{-1} \equiv[\mu(f(q))]^{-1} \equiv s \mu(f(q)) \equiv \mu\left(s^{-1} f(q)\right) \equiv \mu(\alpha(\sigma) f(q)) \equiv$ $\mu(f(\sigma q)) \equiv c$ и $1-c \equiv 1-\mu(f(q)) \equiv t \mu(f(q)) \equiv \mu\left(t^{-1} f(q)\right) \equiv \mu(\alpha(\tau) f(q)) \equiv$ $\mu(f(\tau q)) \equiv c$.

Следствие 5. Эквивариантные эндоморфизмы пространства $\mathcal{C}_{o}^{n}(X)$ индуиируют симплициальные эндоморфизмы комплекса $\mathscr{C}\left(\mathcal{C}_{o}^{n}(X)\right)$, сохраняющие размерности симплексов.

Лемма 11. Если $f$ - эквивариантный эндоморфизм пространства $\mathcal{C}_{o}^{n}(X) u$ $\alpha-$ связанный с ним автоморфизм группь $\mathbf{S}(n)$, mо $f^{*}: \mathscr{C}\left(\mathcal{C}_{o}^{n}(X)\right) \rightarrow \mathscr{C}\left(\mathcal{C}_{o}^{n}(X)\right)$ эквивариантно:

$$
f^{*}(\sigma \mu)=\alpha^{-1}(\sigma) f^{*}(\mu) \quad \text { для всех } \sigma \in \mathbf{S}(n) \text { и всех } \mu \in \mathcal{V}\left(\mathcal{C}_{o}^{n}(X)\right) ;
$$

стало быть, $f^{*}(\mathbf{S}(n) \mu)=\mathbf{S}(n) f^{*}(\mu)$ для всех $\mu \in \mathcal{V}\left(\mathcal{C}_{o}^{n}(X)\right)$. Кроме того, $f^{*}\left(\mathcal{V}_{s r}\left(\mathcal{C}_{o}^{n}(\mathbb{C})\right)\right) \subseteq \mathcal{V}_{s r}\left(\mathcal{C}_{o}^{n}(\mathbb{C})\right) u f^{*}\left(\mathscr{C}_{s r}\left(\mathcal{C}_{o}^{n}(\mathbb{C})\right)\right) \subseteq \mathscr{C}_{s r}\left(\mathcal{C}_{o}^{n}(\mathbb{C})\right)$.

Доказательство. Для $\sigma \in \mathbf{S}(n)$ и $s=\alpha^{-1}(\sigma)$ имеем

$$
\begin{aligned}
{\left[f^{*}(\sigma \mu)\right](q) } & =(\sigma \mu)(f(q))=(\alpha(s) \mu)(f(q))=\mu\left(\alpha\left(s^{-1}\right) f(q)\right) \\
& =\mu\left(f\left(s^{-1} q\right)\right)=\left[f^{*}(\mu)\right]\left(s^{-1} q\right)=\left[s f^{*}(\mu)\right](q)=\left[\alpha^{-1}(\sigma) f^{*}(\mu)\right](q) ;
\end{aligned}
$$

это дает (29). Так как $\alpha^{-1}(\mathbf{S}(n))=\mathbf{S}(n)$, то из (29) следует, что $f^{*}(\mathbf{S}(n) \mu)=$ $\mathbf{S}(n) f^{*}(\mu)$ для $\mu \in \mathcal{V}\left(\mathcal{C}_{o}^{n}(X)\right)$. Согласно леммам 5, 10 и следствию 5, $f^{*}$ симплиииально и сохраняет размерности симплексов. Для $\mu \in \mathcal{V}_{s r}\left(\mathcal{C}_{o}^{n}(\mathbb{C})\right)$ найдется $S R$-чистый $(n-3)$-симплекс $\Delta^{n-3}$ с вершиной $\mu$; так как $\operatorname{dim} f^{*}\left(\Delta^{n-3}\right)=n-3$, то $f^{*}\left(\Delta^{n-3}\right) \notin \mathscr{C}_{c r}\left(\mathcal{C}_{o}^{n}(\mathbb{C})\right)$. Значит, $f^{*}(\nu) \in \mathcal{V}_{s r}\left(\mathcal{C}_{o}^{n}(\mathbb{C})\right)$ хотя бы для одной вершины $\nu \in \Delta^{n-3}$. Конечно, $\mu=\sigma \nu$ для некоторой перестановки $\sigma \in \mathbf{S}(n)$. Согласно $(29), f^{*}(\mu)=f^{*}(\sigma \nu)=\alpha^{-1}(\sigma) f^{*}(\nu)$; поэтому $f^{*}(\mu) \in \mathcal{V}_{s r}\left(\mathcal{C}_{o}^{n}(\mathbb{C})\right)$. Вывод: $f^{*}\left(\mathcal{V}_{s r}\left(\mathcal{C}_{o}^{n}(\mathbb{C})\right)\right) \subseteq \mathcal{V}_{s r}\left(\mathcal{C}_{o}^{n}(\mathbb{C})\right)$. Если $\Delta=\left\{\mu_{0}, \ldots, \mu_{m}\right\} \in \mathscr{C}_{s r}\left(\mathcal{C}_{o}^{n}(\mathbb{C})\right)$, то $\mu_{i} / \mu_{j} \in \mathcal{V}_{s r}\left(\mathcal{C}_{o}^{n}(\mathbb{C})\right)$ при $i \neq j$. Так как $f^{*}$ инъективно на $\Delta$, то $f^{*}\left(\mu_{i}\right) \neq f^{*}\left(\mu_{j}\right)$, $f^{*}\left(\mu_{i}\right) / f^{*}\left(\mu_{j}\right)=f^{*}\left(\mu_{i} / \mu_{j}\right) \in \mathcal{V}_{s r}\left(\mathcal{C}_{o}^{n}(\mathbb{C})\right), f^{*}(\Delta) \in \mathscr{C}_{s r}\left(\mathcal{C}_{o}^{n}(\mathbb{C})\right)$ и $f^{*}\left(\mathscr{C}_{s r}\left(\mathcal{C}_{o}^{n}(\mathbb{C})\right)\right) \subseteq$ $\mathscr{C}_{s r}\left(\mathcal{C}_{o}^{n}(\mathbb{C})\right)$.

Лемма 12. Пусть $n>6-t(X)$ u $f$ - эквивариантный эндоморбизм пространства $\mathcal{C}_{o}^{n}(X)$. Тогда для подходящей перестановки $\sigma \in \mathbf{S}(n)$ и всех $q \in \mathcal{C}_{o}^{n}(X)$ ur $>t(X)$

$$
\begin{array}{ccc}
\frac{f_{r}(\sigma q)-f_{1}(\sigma q)}{f_{2}(\sigma q)-f_{1}(\sigma q)}=\frac{q_{r}-q_{1}}{q_{2}-q_{1}} & \text { nрu } X=\mathbb{C}, \\
\frac{f_{r}(\sigma q)-f_{1}(\sigma q)}{f_{r}(\sigma q)-f_{2}(\sigma q)}: \frac{f_{1}(\sigma q)-f_{3}(\sigma q)}{f_{2}(\sigma q)-f_{3}(\sigma q)}=\frac{q_{r}-q_{1}}{q_{r}-q_{2}}: \frac{q_{1}-q_{3}}{q_{2}-q_{3}} & \text { npu } \quad X=\mathbb{C P}^{1} .
\end{array}
$$

Доказательство. Пусть сначала $X=\mathbb{C}, n>4$ и $\Delta_{s r}^{n-3}$ - первый из трех нормальных ( $n-3)$-симплексов (см. п. 4.6); покажем, что $f^{*}\left(\Delta_{s r}^{n-3}\right) \in \mathbf{S}(n) \Delta_{s r}^{n-3}$. 
Ввиду леммы $9(\mathrm{a})$ достаточно проверить, что нормальный $(n-3)$-симплекс $\Delta_{s r}^{-(n-3)}$ не принадлежит $\mathbf{S}(n) f^{*}\left(\Delta_{s r}^{n-3}\right)$. Пусть $s \in \mathbf{S}(n)$ и $\Delta_{s r}^{-(n-3)}=s f^{*}\left(\Delta_{s r}^{n-3}\right)$. Ясно, что $\left(f_{1}(\sigma q)-f_{i}(\sigma q)\right) /\left(f_{1}(\sigma q)-f_{2}(\sigma q)\right)=\left(q_{1}-q_{2}\right) /\left(q_{1}-q_{i}\right)$ при $i \geqslant 3$, где $\sigma=\alpha(s)$, а $\alpha$ - автоморфизм группы $\mathbf{S}(n)$, отвечающий эндоморфизму $f$. Если $\left(w_{1}-w_{i}\right) /\left(w_{1}-w_{2}\right)=\left(q_{1}-q_{2}\right) /\left(q_{1}-q_{i}\right)$ для всех $i \geqslant 3$, то $s r_{i j k}(w)=c r_{i, j, 1, k}(q)$ для всех попарно различных $i, j, k \geqslant 3$. Значит, $\left[f^{*}\left(s r_{i j k}\right)\right](\sigma q)=s r_{i j k}(f(\sigma q))=$ $c r_{i, j, 1, k}(q)$, что противоречит лемме 11.

Пусть теперь $X=\mathbb{C P}^{1}, n>3, \Delta_{c r}^{n-4}=\left[c r_{1,2,3,4}, c r_{1,2,3,5}, \ldots, c r_{1,2,3, n}\right] \in$ $\mathscr{C}_{c r}\left(\mathbf{q}_{n}\right)$. Согласно леммам 10 и $5, f^{*}\left(\Delta_{c r}^{n-4}\right)$ есть $(n-4)$-мерный симплекс в $\mathscr{C}\left(\mathcal{C}_{o}^{n}\left(\mathbb{C P}^{1}\right)\right)$. По теореме $12 \mathscr{C}\left(\mathcal{C}_{o}^{n}\left(\mathbb{C P}^{1}\right)\right)=\mathscr{C}_{c r}\left(\mathbf{q}_{n}\right)$; значит, $f^{*}\left(\Delta_{c r}^{n-4}\right) \in \mathscr{C}_{c r}^{n-4}\left(\mathbf{q}_{n}\right)$. По лемме $9(\mathrm{~b})$ действие группы $\mathbf{S}(n)$ на $\mathscr{C}_{c r}^{n-4}\left(\mathbf{q}_{n}\right)$ транзитивно, так что $f^{*}\left(\Delta_{c r}^{n-4}\right)$ $\in \mathbf{S}(n) \Delta_{c r}^{n-4}$.

Замечание 5. (а) Из леммы 12 следует, что $f^{*}\left(\mathcal{V}_{c r}\left(\mathcal{C}_{o}^{n}(\mathbb{C})\right)\right)=\mathcal{V}_{c r}\left(\mathcal{C}_{o}^{n}(\mathbb{C})\right)$.

(b) При $n>t(X)$ существуют непустые открытые по Зарискому множества $U \subset \mathcal{C}_{o}^{n}(X)$ и $V \subset \mathcal{C}^{n}(X)$, такие, что: (1) если $q \in U, A \in \operatorname{Aut} X, s \in \mathbf{S}(n)$ и $A q=s q$, то $A=$ id и $s=1 ;(2)$ если $Q \in V, A \in \operatorname{Aut} X$ и $A Q=Q$, то $A=$ id. И правда, $\left\{q \in \mathcal{C}_{o}^{n}(X) \mid s q=A q\right.$ для некоторых $s \in \mathbf{S}(n) \backslash\{1\}$ и $A \in$ Aut $\left.X\right\}-$ собственное замкнутое по Зарискому подмножество в $\mathcal{C}_{o}^{n}(X)$; это дает (1) и (2).

4.7. Доказательство главной части теоремы 4 для $X=\mathbb{C}_{\text {и }} \mathbb{C P}^{1}$. Мы докажем, что при $n>4$ всякий неабелев эндоморфизм $F$ пространства $\mathcal{C}^{n}(X)$ является ручным. Пусть $f$ - эквивариантный эндоморфизм пространства $\mathcal{C}_{o}^{n}(X)$, накрывающий $F$ (см. теорему 11 ); достаточно проверить, что $f$ ручной.

Теорема 13. При $n>6-t(X)$ каждый эквивариантный эндоморфизм $f$ пространства $\mathcal{C}_{o}^{n}(X)$ является ручным.

Доказательство. По лемме 12 существует такая перестановка $\sigma \in \mathbf{S}(n)$, что для всех $q \in \mathcal{C}_{o}^{n}(X)$ и всех $r>t(X)$ при $X=\mathbb{C}$ выполняется $\left(16^{*}\right)$, а при $X=$ $\mathbb{C P}^{1}$ выполняется $\left(17^{*}\right)$. Определим морфизм $\tau: \mathcal{C}_{o}^{n}(X) \rightarrow$ Aut $X$ формулами

$$
\begin{array}{cl}
\frac{f_{1}(\sigma q)-\tau(q) x}{f_{1}(\sigma q)-f_{2}(\sigma q)}=\frac{q_{1}-x}{q_{1}-q_{2}} & \text { при } x \in X=\mathbb{C} ; \\
\frac{\tau(q) x-f_{1}(\sigma q)}{\tau(q) x-f_{2}(\sigma q)}: \frac{f_{1}(\sigma q)-f_{3}(\sigma q)}{f_{2}(\sigma q)-f_{3}(\sigma q)}=\frac{x-q_{1}}{x-q_{2}}: \frac{q_{1}-q_{3}}{q_{2}-q_{3}} & \text { при } x \in X=\mathbb{C P}^{1} .
\end{array}
$$

Согласно лемме $12, \tau(q) q_{i}=f_{i}(\sigma q)$ при $i=1, \ldots, n$ и $q=\left(q_{1}, \ldots, q_{n}\right) \in \mathcal{C}_{o}^{n}(X)$. Следовательно, $\tau(q) q=f(\sigma q)=\alpha(\sigma) f(q)$, т. е. $f(q)=\alpha\left(\sigma^{-1}\right) \tau(q) q$ для всех $q \in \mathcal{C}_{o}^{n}(X)$. Далее, $s \tau(s q) q=\tau(s q) s q=f(\sigma s q)=\alpha(\sigma s) f(q)=\alpha(\sigma s) f\left(\sigma \sigma^{-1} q\right)=$ $\alpha(\sigma s) \tau\left(\sigma^{-1} q\right) \sigma^{-1} q=\alpha(\sigma s) \sigma^{-1} \tau\left(\sigma^{-1} q\right) q$ для $s \in \mathbf{S}(n)$ и $q \in \mathcal{C}_{o}^{n}(X)$, откуда $\left[(\tau(s q))^{-1} \cdot \tau\left(\sigma^{-1} q\right)\right] q=\sigma \alpha\left(s^{-1} \sigma^{-1}\right) s q(« \bullet »-$ умножение в Aut $X)$. Согласно замечанию $5(\mathrm{~b})$, это дает $\sigma \alpha\left(s^{-1} \sigma^{-1}\right) s=1$ и $\tau(s q)=\tau\left(\sigma^{-1} q\right)$ для всех $s \in \mathbf{S}(n)$ и всех $q$ из непустого открытого по Зарискому множества $U \subset \mathcal{C}_{o}^{n}(X)$. По непрерывности $\tau(s q)=\tau\left(\sigma^{-1} q\right)$ для всех $q \in \mathcal{C}_{o}^{n}(X)$ и $s \in \mathbf{S}(n)$, что дает $\mathbf{S}(n)$-инвариантность морфизма $\tau$.

Замечание 6. Так как $\sigma \alpha\left(s^{-1} \sigma^{-1}\right) s=1$ для всех $s \in \mathbf{S}(n)$, то автоморфизм $\alpha$ внутренний; для $n \neq 6$ это уже было отмечено в доказательстве теоремы 11. 


\section{8. Стабилизаторы точек и размерность образа неабелева эндомор- физма пространства $\mathcal{C}^{n}(X)$.}

Лемма 13. При $n \geqslant t(X)$ стабилизатор $\operatorname{St}_{Q}=\{A \in \operatorname{Aut} X \mid A Q=Q\}$ точки $Q=\left\{q_{1}, \ldots, q_{n}\right\} \in \mathcal{C}^{n}(X)$ либо является конечной разрешимой группой, либо изоморбен $\mathbf{A}(5) ;$ для точек $Q$ общего положения \# $\operatorname{St}_{Q}=\{1\}$.

Доказательство. Любой элемент $A \in \mathrm{St}_{Q}$ - преобразование Мёбиуса, переставляющее точки $q_{1}, \ldots, q_{n} \in X$. Оно однозначно определяется своими значениями $A q_{1}, \ldots, A q_{t(X)}$, так что $\# \mathrm{St}_{Q}<\infty$; для точек общего положения $\# \mathrm{St}_{Q}=\{1\}$.

Если $X=\mathbb{C}$, то подгруппа $\mathrm{St}_{Q} \subset \mathbf{A f f} \mathbb{C}$ сопряжена конечной подгруппе максимальной компактной подгруппы $\mathbb{T} \subset \mathbf{A f f} \mathbb{C}$, состоящей из всех вращений $z \mapsto e^{i t} z, t \in \mathbb{R}$; значит, $\mathrm{St}_{Q}$ - конечная циклическая группа.

Максимальные компактные подгруппы в $\mathbf{P S L}(2, \mathbb{C})$ сопряжены группе преобразований Мёбиуса вида $z \mapsto(a z-\bar{c}) /(c z+\bar{a}),|a|^{2}+|c|^{2}=1$, изоморфной $\mathbf{S O}(3)$. Конечные подгруппы группы $\mathbf{S O}(3)$ известны со времен Ф. Клейна (см., например, [36] или [22, гл. VI, §§51-57]); все они, за исключением групп вращений икосаэдра и додекаэдра, изоморфных $\mathbf{A}(5)$, разрешимы.

Из теоремы 13 следует, что при $n>4$ все (Aut $X$ )-орбиты в $\mathcal{C}^{n}(X)$ инвариантны относительно неабелевых эндоморфизмов $F: \mathcal{C}^{n}(X) \rightarrow \mathcal{C}^{n}(X)$. Поэтому $\operatorname{dim}_{\mathbb{C}} F\left(\mathcal{C}^{n}(X)\right) \geqslant n-t(X)$. Эту оценку можно усилить, доведя до неулучшаемой:

Теорема 14. Eсли $n>4$, mo $\operatorname{dim}_{\mathbb{C}} F\left(\mathcal{C}^{n}(X)\right) \geqslant n-t(X)+1$.

Это следует из доказанной в п. 4.7 части теоремы 4, наличия точек $Q \in \mathcal{C}^{n}(X)$ с $\operatorname{St}_{Q} \neq\{1\}$ (скажем, $\left.Q=\left\{1, e^{2 \pi i / n}, \ldots, e^{2 \pi i(n-1) / n}\right\}\right)$ и следующей леммы.

Лемма 14. Пусть $U \subseteq \mathcal{C}^{n}(X)$ непусто, открыто и (Aut $X$ )-инвариантно, отображение $T: U \rightarrow$ Aut $X$ голоморбно, $F: U \rightarrow \mathcal{C}^{n}(X), F(Q) \stackrel{\text { def }}{=} T(Q) Q$. Если $Q^{*} \in U$ и $\operatorname{St}_{Q^{*}} \neq\{1\}$, то найдется такое непустое открытое (Aut $X$ ) инвариантное множество $U^{\circ} \subseteq U$, что для всякого $Q \in U^{\circ}$ в $F(($ Aut $X) Q)$ есть подмножсетво, изоморбное диску $\mathbb{D} \subset \mathbb{C}$. Стало бъьть, $\operatorname{dim}_{\mathbb{C}} F\left(U^{\circ}\right) \geqslant$ $n-t(X)+1$.

Доказательство. $\operatorname{dim}_{\mathbb{C}}[(\operatorname{Aut} X) Q]=\operatorname{dim}_{\mathbb{C}}($ Aut $X)=t(X)$ для всех $Q \in U$. Рассмотрим морфизм $f_{Q}:$ Aut $X \rightarrow($ Aut $X) Q, f_{Q}(A) \stackrel{\text { def }}{=} T(A Q) A Q=F(A Q)$. Его дифференциал $\left(d f_{Q^{\circ}}\right)\left(A^{\circ}\right): \mathbb{C}^{t(X)} \rightarrow \mathbb{C}^{t(X)}$ для некоторых $Q^{\circ} \in U$ и $A^{\circ} \in$ Aut $X$ отличен от нуля. В самом деле, иначе ввиду связности Aut $X$ получаем $f_{Q}=$ const при каждом $Q \in U$, т. е. $T(A Q) A Q \equiv T(Q) Q$ и $(T(Q))^{-1} T(A Q) A \in$ $\mathrm{St}_{Q}$ для $(Q, A) \in U \times($ Aut $X)$; так как $\# \mathrm{St}_{Q}<\infty$, то $(T(Q))^{-1} T(A Q) A$ не зависит от $A$, и $(T(Q))^{-1} T(A Q) A=(T(Q))^{-1} T(Q)=1$ при $(Q, A) \in U \times($ Aut $X)$. Итак, $A=\left(T\left(Q^{*}\right)\right)^{-1} T\left(Q^{*}\right) A=\left(T\left(Q^{*}\right)\right)^{-1} T\left(A Q^{*}\right) A=1$ для всякого $A \in \operatorname{St}_{Q^{*}}$ вопреки условию $\operatorname{St}_{Q^{*}} \neq\{1\}$.

Пусть $\left(Q^{\circ}, A^{\circ}\right) \in U \times($ Aut $X)$ и $\left(d f_{Q^{\circ}}\right) A^{\circ} \neq 0$, т. е. $\operatorname{rank}\left[\left(d f_{Q^{\circ}}\right)\left(A^{\circ}\right)\right] \geqslant 1$. По полунепрерывности $\operatorname{rank}\left[\left(d f_{Q}\right)(A)\right] \geqslant 1$ в окрестности $U^{\circ} \times \mathcal{A}^{\circ}$ точки $\left(Q^{\circ}, A^{\circ}\right)$, и для $Q \in U^{\circ}$ образ (Aut $\left.X\right)$-орбиты $F(($ Aut $X) Q)=f_{Q}($ Aut $X)$ содержит множество, изоморфное диску $\mathbb{D}$, так что $\operatorname{dim}_{\mathbb{C}} F\left(U^{\circ}\right) \geqslant n-t(X)+1$. 
Замечание 7. Вот более информативная версия теоремы 14 для $X=\mathbb{C}$. Проектор $L=\left(L_{1}, \ldots, L_{n}\right), L_{i}\left(\left\{q_{1}, \ldots, q_{n}\right\}\right)=q_{i}-\left(q_{1}+\cdots+q_{n}\right) / n$, отображает $\mathcal{C}^{n}(\mathbb{C})$ на пространство $\mathcal{C}_{b}^{n-1}(\mathbb{C})=\left\{Q \in \mathcal{C}^{n}(\mathbb{C}) \mid \sum_{q \in Q} q=0\right\}$ полиномов с простыми корнями и старшими коэффициентами 1 и 0 . Если $n>4 u$ $F: \mathcal{C}_{b}^{n-1}(\mathbb{C}) \rightarrow \mathcal{C}^{n}(\mathbb{C})$ - неабелев морфизм, то $L\left(F\left(\mathcal{C}_{b}^{n-1}(\mathbb{C})\right)\right)=\mathcal{C}_{b}^{n-1}(\mathbb{C})$. Пусть $F^{\prime}=F \circ L ;$ по теореме $4 F^{\prime}(Q)=T(Q) Q$ с голоморфным $T: \mathcal{C}^{n}(\mathbb{C}) \rightarrow \operatorname{Aff} \mathbb{C}$, т. е. $[T(Q)](z)=A(Q) z+B(Q)$ при $Q \in \mathcal{C}^{n}(\mathbb{C})$ и $z \in \mathbb{C}$, где $A: \mathcal{C}^{n}(\mathbb{C}) \rightarrow \mathbb{C}^{*}$ и $B: \mathcal{C}^{n}(\mathbb{C}) \rightarrow \mathbb{C}$ голоморфны. Класс когомологий $d_{n}^{*} \in H^{1}\left(\mathcal{C}^{n}(\mathbb{C}), \mathbb{Z}\right) \cong \mathbb{Z}$ дискриминанта $d_{n}: \mathcal{C}^{n}(\mathbb{C}) \rightarrow \mathbb{C}^{*}$ порождает $H^{1}\left(\mathcal{C}^{n}(\mathbb{C}), \mathbb{Z}\right)$, так что $A=d_{n}^{r} e^{\varphi}$, где $r \in \mathbb{Z}$ и $\varphi$ голоморфна на $\mathcal{C}^{n}(\mathbb{C})$. Для $Q^{\circ}=\left\{q_{1}^{\circ}, \ldots, q_{n}^{\circ}\right\} \in \mathcal{C}_{b}^{n-1}(\mathbb{C})$ голоморфная функция $a: \mathbb{C}^{*} \ni \zeta \mapsto a(\zeta)=\zeta A\left(\zeta Q^{\circ}\right) \in \mathbb{C}^{*}$ непостоянна, ибо $a(\zeta)=\zeta A\left(\zeta Q^{\circ}\right)=$ $\zeta \cdot d_{n}^{r}\left(\zeta Q^{\circ}\right) e^{\varphi\left(\zeta Q^{\circ}\right)}=\zeta^{r n(n-1)+1} d_{n}^{r}\left(Q^{\circ}\right) e^{\varphi\left(\zeta Q^{\circ}\right)}$. По теореме Пикара $a\left(\zeta^{\circ}\right)=1$ для некоторого $\zeta^{\circ} \in \mathbb{C}^{*}$ и $L_{i}\left(F\left(\zeta^{\circ} Q^{\circ}\right)\right)=\zeta^{\circ} A\left(\zeta^{\circ} Q^{\circ}\right) q_{i}^{\circ}=a\left(\zeta^{\circ}\right) q_{i}^{\circ}=q_{i}^{\circ}$ для всех $i$, т. е. $Q^{\circ} \in L\left(F\left(\mathcal{C}_{b}^{n-1}(\mathbb{C})\right)\right)$.

4.9. Абелевы морфизмы. Нам понадобятся следующие две леммы.

Лемма 15. Если $n>4, k \geqslant t(X)$ u $Q \in \mathcal{C}^{k}(X)$, то каждый гомоморфизм $\varphi: B_{n}(X) \rightarrow \pi_{1}((\operatorname{Aut} X) Q)$ абелев.

Доказательство. Если $\operatorname{St}_{Q}=\{1\}$, то $($ Aut $X) Q \cong$ Aut $X$ и $\pi_{1}(($ Aut $X) Q) \cong$ $\pi_{1}($ Aut $X)$ - циклическая группа, так что все ясно.

Пусть $\operatorname{St}_{Q} \neq\{1\}$. Тогда $h:$ Aut $X \rightarrow($ Aut $X) Q$ - конечное накрытие со слоем $\mathrm{St}_{Q} ;$ возникает коммутативная диаграмма с точной строкой

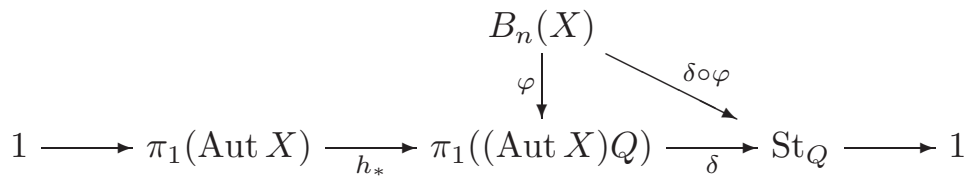

Достаточно убедиться, что $\delta \circ \varphi$ абелев, ибо тогда $(\delta \circ \varphi)\left(B_{n}^{\prime}(X)\right)=\{1\}$, группа $\varphi\left(B_{n}^{\prime}(X)\right) \subseteq \operatorname{Ker} \delta=h_{*}\left(\pi_{1}(\right.$ Aut $\left.X)\right) \cong \pi_{1}($ Aut $X)$ абелева, $\varphi\left(B_{n}^{\prime}(X)\right)=\{1\}$ и $\varphi$ абелев. По лемме 13 группа $\mathrm{St}_{Q}$ либо разрешима, либо изоморфна $\mathbf{A}(5)$. В первом случае все ясно; пусть $\mathrm{St}_{Q} \cong \mathbf{A}(5)$. Если $n>5$, то по теореме 8 гомоморфизм $\delta \circ \varphi$ абелев; если же $n=5$, то $(\delta \circ \varphi)\left(B_{5}(X)\right) \subseteq \mathbf{A}(5) \varsubsetneqq \mathbf{S}(5)-$ собственная подгруппа в $\mathbf{S}(5)$ и, согласно теореме $8, \delta \circ \varphi$ абелев.

Лемма 16. Пусть комплексное пространство $Z$ лиувиллево 1), и пусть отображсение $F: Z \rightarrow \mathcal{C}^{m}(X)$ голоморфно. Если подгруппа $F_{*}\left(\pi_{1}(Z)\right) \subseteq \pi_{1}\left(\mathcal{C}^{m}(X)\right)$ разрешима, то $F(Z)$ содержится в одной орбите (Aut $X)$-действия в $\mathcal{C}^{m}(X)$.

Доказательство. Пусть $f: Y \rightarrow \mathcal{C}_{o}^{m}(X)$ - голоморфное поднятие $F$ на подходящее связное конечное накрытие $Y$ над $Z$; конечно, $Y$ лиувиллево. Учитывая изоморфизм $j_{X, m}$ (см. $\left.\S 3\right)$, получим цепочку голоморфных отображений

$$
H: Y \stackrel{f}{\rightarrow} \mathcal{C}_{o}^{m}(X) \stackrel{j_{X, m}}{\longrightarrow}(\operatorname{Aut} X) \times \mathcal{C}_{o}^{m-t(X)}(\mathbb{C} \backslash\{0,1\}) \stackrel{\xi}{\rightarrow} \mathcal{C}_{o}^{m-t(X)}(\mathbb{C} \backslash\{0,1\}),
$$

где $\xi$ - естественная проекция. Покажем, что $H=$ const; тогда $f(Y) \subseteq($ Aut $X) q$ для некоторого $q \in \mathcal{C}_{o}^{m}(X)$ и $F(Z) \subseteq($ Aut $X) Q$, где $Q=p(q)$ - проекция точки

1) То есть на нем нет непостоянных ограниченных голоморфных функций. Все используемые в данной работе свойства лиувиллевых пространств элементарны и хорошо известны; более интересные вещи, связанные с понятием лиувиллевости, можно найти в [44], [47]. 
$q$ на $\mathcal{C}^{m}(X)$. Каждая координата $h: Y \rightarrow \mathbb{C} \backslash\{0,1\}$ отображения $H$ голоморфна. Подгруппа $\Gamma \stackrel{\text { def }}{=} h_{*}\left(\pi_{1}(Y)\right) \subseteq \pi_{1}(\mathbb{C} \backslash\{0,1\}) \cong \mathbb{F}_{2}$ разрешима; значит, $\Gamma=\{1\}$ или $\Gamma \cong \mathbb{Z}$. Пусть $V \rightarrow \mathbb{C} \backslash\{0,1\}$ - накрытие, отвечающее $Г$. Тогда $V$ - диск или кольцо, так что отображение $\widetilde{h}: Y \rightarrow V$, накрывающее $h$, постоянно; значит, $h=$ const и $H=$ const.

Каждая связная комплексная группа Ли $\mathcal{L}$ лиувиллева (ибо в ней много однопараметрических подгрупп), а группа $\pi_{1}(\mathcal{L})$ абелева; поэтому образ любого голоморфного отображения $\mathcal{L} \rightarrow \mathcal{C}^{m}(X)$ содержится в одной (Aut $X$ )-орбите.

Следствие 6. Морфизмы $F: \mathcal{C}^{n}(X) \rightarrow \mathcal{C}^{k}(X)$ отображсают (Aut $X$ )-орбитъ в (Aut $X)$-орбить.

Следующая теорема завершает доказательство теоремы 4 для $X=\mathbb{C P}^{1}$ или $\mathbb{C}$.

Теорема 15. Пусть $X$ есть $\mathbb{C P}^{1}$ или $\mathbb{C}, n>4 u k \geqslant t(X)=\operatorname{dim}_{\mathbb{C}}($ Aut $X)$. Тогда всякий абелев морфизм $F: \mathcal{C}^{n}(X) \rightarrow \mathcal{C}^{k}(X)$ одноорбитен, а каждое непрерывное одноорбитное отображсение $F: \mathcal{C}^{n}(X) \rightarrow \mathcal{C}^{k}(X)$ абелево.

Доказательство. Первое утверждение следует из леммы 16, ибо $\mathcal{C}^{n}(X)$, как всякое связное квазипроективное многообразие, лиувиллево, а $F_{*}\left(\pi_{1}\left(\mathcal{C}^{n}(X)\right)\right)-$ по условию абелева группа. Второе утверждение вытекает из леммы 15.

Замечание 8. Если $n \neq 4$ и $n(n-1)$ не делит $k(k-1)$, то каждый морфизм $\mathcal{C}^{n}(\mathbb{C}) \rightarrow \mathcal{C}^{k}(\mathbb{C})$ абелев и потому одноорбитен (см. [43, п. 8.1] и [50, §9]). Я не знаю, существуют ли неабелевы морфизмы $\mathcal{C}^{n}(\mathbb{C}) \rightarrow \mathcal{C}^{k}(\mathbb{C})$ при $k>n>4$.

4.10. Автоморфизмы пространства $M_{o}(\mathbf{0}, \boldsymbol{n})^{1)}$. Пространство модулей $M_{o}(0, n)$ римановой сферы с $n$ упорядоченными проколами изоморфно пространству орбит $\mathcal{C}_{o}^{n}\left(\mathbb{C P}^{1}\right) / \mathbf{P S L}(2, \mathbb{C})$, которое, как мы знаем, есть $\mathcal{D}^{n-3}\left(\mathbb{C P}^{1}\right)=$ $\left\{q \in \mathcal{C}_{o}^{n}\left(\mathbb{C P}^{1}\right) \mid q=\left(q_{1}, \ldots, q_{n-3}, 0,1, \infty\right)\right\} \cong \mathcal{C}_{o}^{n-3}(\mathbb{C} \backslash\{0,1\})$ (§3). Для $h=$ $\left(h_{1}, \ldots, h_{n-3}\right): \mathcal{D}^{n-3}\left(\mathbb{C P}^{1}\right) \rightarrow \mathcal{D}^{n-3}\left(\mathbb{C P}^{1}\right)$ положим $h_{n-2}=0, h_{n-1}=1, h_{n}=\infty$. Калиман [29] обнаружил следующий факт:

Теорема 16. Пусть $n \geqslant 4 u h=\left(h_{1}, \ldots, h_{n-3}\right)$ - непостоянный эндоморфизм пространства $\mathcal{D}^{n-3}\left(\mathbb{C P}^{1}\right)$. Тогда

$$
h_{i}(q)=\frac{q_{\sigma(i)}-q_{\sigma(n-2)}}{q_{\sigma(i)}-q_{\sigma(n)}}: \frac{q_{\sigma(n-2)}-q_{\sigma(n-1)}}{q_{\sigma(n)}-q_{\sigma(n-1)}}
$$

при всех $i=1, \ldots, n-3$, где $\sigma \in \mathbf{S}(n)$ не зависит от $i . B$ частности, $h-$ автоморфизм.

В [29] нет доказательства; вот его упрощенная версия в духе пп. 4.3-4.7.

Доказательство. Все $h_{i}: \mathcal{D}^{n-3}\left(\mathbb{C P}^{1}\right) \rightarrow \mathbb{C} \backslash\{0,1\}$ с $i \leqslant n-3$ голоморфны; покажем, что $h_{i} \neq$ const. Допустим, что $h_{p}=C=$ const $\neq 0,1$ для какогонибудь $p \leqslant n-3$; так как $h \neq$ const, то $h_{r} \neq$ const для некоторого $r \leqslant n-3$. Так как $h_{r}(q) \neq h_{p}(q)$ для всех $q \in \mathcal{D}^{n-3}\left(\mathbb{C P}^{1}\right)$, то $h_{r}(q) \neq 0,1, C$ в $\mathcal{D}^{n-3}\left(\mathbb{C P}^{1}\right)$. Согласно замечанию $4, h_{r}=\left(q_{i}-q_{j}\right)\left(q_{k}-q_{l}\right) /\left(q_{i}-q_{k}\right)\left(q_{i}-q_{k}\right)$, где $i, j, k, l \in$ $\{1, \ldots, n\}$ попарно различны; но такая функция на $\mathcal{D}^{n-3}\left(\mathbb{C P}^{1}\right)$ принимает все значения $a \in \mathbb{C} \backslash\{0,1\}$, включая и $C$, и мы приходим к противоречию.

1) На вопрос об автоморфизмах пространства $M_{o}(0, n)$, поставленный, кажется, У. Фултоном, мое внимание обратил И. В. Долгачев. 
Рассмотрим теперь композицию

$$
\Phi: \mathcal{C}_{o}^{n}\left(\mathbb{C P}^{1}\right) \stackrel{j_{\mathbb{C P}^{1}, n}}{\longrightarrow} \operatorname{PSL}(2, \mathbb{C}) \times \mathcal{D}^{n-3}\left(\mathbb{C P}^{1}\right) \stackrel{\nu}{\longrightarrow} \mathcal{D}^{n-3}\left(\mathbb{C P}^{1}\right) \stackrel{h}{\longrightarrow} \mathcal{D}^{n-3}\left(\mathbb{C P}^{1}\right),
$$

где $\nu$ - естественная проекция. Пусть $f_{i}(q)=q_{i}$ для всех $q=\left(q_{1}, \ldots, q_{n}\right)$ и $i \leqslant$ $n-3$; тогда $\Delta^{n-4}=\left\{f_{1}, \ldots, f_{n-3}\right\} \in \mathscr{C}\left(\mathcal{D}^{n-3}\left(\mathbb{C P}^{1}\right)\right)$. Так как $h-$ эндоморфизм пространства $\mathcal{D}^{n-3}\left(\mathbb{C P}^{1}\right)$ и $h_{1}, \ldots, h_{n-3} \neq$ const, то $h^{*}\left(\Delta^{n-4}\right)=\left\{h_{1}, \ldots, h_{n-3}\right\} \in$ $\mathscr{C}\left(\mathcal{D}^{n-3}\left(\mathbb{C P}^{1}\right)\right)$ и $\Phi^{*}\left(\Delta^{n-4}\right)=j_{\mathbb{C P}^{1}, n}^{*}\left(\nu^{*}\left(h^{*}\left(\Delta^{n-4}\right)\right)\right) \in \mathscr{C}\left(\mathcal{C}_{o}^{n}\left(\mathbb{C P}^{1}\right)\right)$. По лемме $9(\mathrm{~b})$ подходящая перестановка координат $q_{1}, \ldots, q_{n}$ в $\mathcal{C}_{o}^{n}\left(\mathbb{C P}^{1}\right)$ приводит $\Phi^{*}\left(\Delta^{n-4}\right)$ к виду $\Delta_{c r}^{m}$ с $m=n-4$ (см. п. 4.6). С точностью до обозначений, это и есть форма $(*)$. Наконец, всякое отображение $h$ с компонентами вида $(*)-$ автоморфизм пространства $\mathcal{D}^{n-3}\left(\mathbb{C P}^{1}\right)$.

Так как $\sigma A q=A \sigma q$ при $\sigma \in \mathbf{S}(n), A \in \mathbf{P S L}(2, \mathbb{C})$ и $q \in \mathcal{C}_{o}^{n}\left(\mathbb{C P}^{1}\right)$, то перестановка $\sigma \in \mathbf{S}(n)$ порождает автоморфизм пространства $M_{o}(0, n)$. Из теоремы 16 получаем

Следствие 7. Каждый непостоянный эндоморфизм пространства модулей $M_{o}(0, n)$ является автоморфизмом и порождается некоторой перестановкой координат в $\mathcal{C}_{o}^{n}\left(\mathbb{C P}^{1}\right)$, maж ито Aut $M_{o}(0, n) \cong \mathbf{S}(n)$.

\section{§5. Точные представления суперпозициями}

Теорема 17. При $n>4$ функиия $u_{n}$ не имеет точного представления суперпозичиями целых алгеброидных функций от менее чем $n-1$ переменных и голоморфных функиий любого числа переменных.

Доказательство. Поскольку конечное накрытие с примитивной группой монодромии неразложимо, суперпозиция, представляющая $u_{n}$, содержит ровно одну многозначную функцию, т. е.

$$
u_{n}(z)=P(\varphi(h(z)), z) .
$$

Здесь $h: \mathbb{C}^{n} \rightarrow \mathbb{C}^{k}$ и $P: \mathbb{C}^{n+1} \rightarrow \mathbb{C}-$ голоморфные отображения, а $\varphi-$ целая $n$-значная алгеброидная функция на $\mathbb{C}^{k}$, т.е. решение уравнения $\varphi^{n}+$ $g_{1}(w) \varphi^{n-1}+\cdots+g_{n}(w)=0$, где $w \in \mathbb{C}^{k}$ и отображение $g=\left(g_{1}, \ldots, g_{n}\right): \mathbb{C}^{k} \rightarrow \mathbb{C}^{n}$ голоморфно. Пусть $\Sigma_{\varphi}$ - дискриминантное множество функции $\varphi$. Формула $(*)$ приводит к коммутативной диаграмме

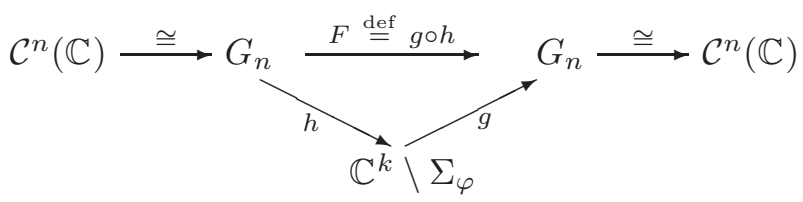

из которой следует, что $\operatorname{dim}_{\mathbb{C}} F\left(\mathcal{C}^{n}(\mathbb{C})\right) \leqslant k$. Морфизм $F$ неабелев, ибо иначе абелевыми оказались бы группы монодромии функций $\varphi(h(z))$ и $u_{n}(z)=$ $P(\varphi(h(z)), z)$; по теореме $14 k \geqslant \operatorname{dim}_{\mathbb{C}} F\left(\mathcal{C}^{n}(\mathbb{C})\right) \geqslant n-2+1=n-1$.

Целые алгебраические функции общего положения вовсе не имеют точных представлений суперпозициями целых алгебраических функций меньшего числа переменных. Пусть $\mathcal{E} \mathcal{A}(n, k)$ - класс всех $n$-значных целых алгебраических функций на $\mathbb{C}^{k}$. Все $g \in \mathcal{E} \mathcal{A}(n, k)$ являются суперпозициями целых алгебраических функций от $n-1$ переменных; поэтому будем с самого начала считать, 
что $k<n$. Отождествляя каждую функцию $g$ с вектором всех коэффициентов ее определяющего полинома $R_{g}$, превратим множество $\mathcal{E} \mathcal{A}^{N}(n, k)$ всех $g \in \mathcal{E} \mathcal{A}(n, k)$, у которых все коэффициенты полинома $R_{g}$ - полиномы степени $\leqslant N$, в конечномерное векторное пространство. Подмножество $A \subset \mathcal{E} \mathcal{A}(n, k)$ назовем тонким, если для каждого $N$ пересечение $A \cap \mathcal{E} \mathcal{A}^{N}(n, k)$ содержится в собственном алгебраическом подмножестве пространства $\mathcal{E} \mathcal{A}^{N}(n, k)$.

Теорема 18. При $n>4 u k<n$ множество всех $n$-значных иельх алгебраических функиий от $k$ переменных, точно представимых суперпозициями целых алгебраических функиий от менее чем $k$ переменных, является тонким подмножеством в $\mathcal{E} \mathcal{A}(n, k)$.

Идея доказательства такова (см. [42]). Пусть $\mathcal{O}_{V}$ - пучок ростков голоморфных функций в области $V \subseteq \mathbb{C}^{k}, \mathcal{O}(V)$ - кольцо голоморфных функций на $V, t=g(v)$ - целая алгеброидная функция в $V$, определяемая уравнением вида $R(t, v)=t^{n}+a_{1}(v) t^{n-1}+\cdots+a_{n}(v)=0, a_{i} \in \mathcal{O}(V)$, и пусть $\mathbf{R}_{v}-$ росток полинома $R$ в точке $v \in V$. Пусть $\Sigma_{g}(V, r) \subseteq V(2 \leqslant r \leqslant n)$ состоит из всех $v \in V$, таких, что $R(t, v)$ имеет корень кратности $\geqslant r$; конечно, $\Sigma_{g}(V, 2)=\Sigma_{g}$ - дискриминантное множество функции $g$ в $V$. Пусть $\Sigma_{g}^{*}(V, r)$ - множество всех тех $v \in V$, для которых $\mathbf{R}_{v}$ имеет неприводимый делитель степени $\geqslant r$ в кольце $\mathcal{O}_{v}[t]$; тогда $\Sigma_{g}(V, r) \supseteq \Sigma_{g}^{*}(V, r)$ и $\Sigma_{g} \supseteq \Sigma_{g}^{*}(V, 2) \supseteq \ldots \supseteq$ $\Sigma_{g}^{*}(V, n)$. Пусть $\operatorname{brm}(g, V)=\max \left\{r \mid \Sigma_{g}^{*}(V, r) \neq \varnothing\right\} \quad\left(\right.$ при $\Sigma_{g}^{*}(V, 2)=\varnothing$ считаем, что $\operatorname{brm}(g, V)=1)$. В [42] доказана следующая теорема (теорема 2.5): пусть $\Sigma_{g} \neq V$, группа монодромии $\operatorname{Gal}(g, V)$ примитивна, $r=\operatorname{brm}(g, V) \geqslant 2$, u пусть $d_{r}=\operatorname{dim}_{\mathbb{C}} \Sigma_{g}(V, r) ;$ тогда функиия $g$ в области $V$ не может быть точно представлена суперпозичиями иельх алгеброидных функиий от менее чем $k-d_{r}$ переменных.

Определим множества $A_{1}, A_{2}, A_{3}, A_{4}$ так: (а) $A_{1}$ - все $g \in \mathcal{E} \mathcal{A}^{N}(n, k)$ с $\Sigma_{g}=\mathbb{C}^{k} ;$ (b) $A_{2}$ - все $g \in \mathcal{E} \mathcal{A}^{N}(n, k) \backslash A_{1}$ с $\mathbf{G a l}\left(g, \mathbb{C}^{k}\right) \neq \mathbf{S}(n) ;$ (c) $A_{3}$ - все $g \in$ $\mathcal{E} \mathcal{A}^{N}(n, k) \backslash\left(A_{1} \cup A_{2}\right)$ с $\operatorname{brm}\left(g, \mathbb{C}^{k}\right)<k+1$; (d) $A_{4}$ - все $g \in \mathcal{E} \mathcal{A}^{N}(n, k) \backslash\left(A_{1} \cup A_{2} \cup\right.$ $\left.A_{3}\right)$ с $d_{k+1} \stackrel{\text { def }}{=} \operatorname{dim}_{\mathbb{C}} \Sigma_{g}\left(\mathbb{C}^{k}, k+1\right)>0$. Ясно, что $A_{1}$ - собственное алгебраическое подмножество в $\mathcal{E} \mathcal{A}^{N}(n, k)$. Оказывается, $A_{2}, A_{3}, A_{4}$ содержатся в собственных алгебраических подмножествах пространства $\mathcal{E} \mathcal{A}^{N}(n, k)$; поэтому таково и $A=$ $A_{1} \cup A_{2} \cup A_{3} \cup A_{4}$. Для всех $g \in \mathcal{E} \mathcal{A}^{N}(n, k) \backslash A$ выполняются следующие условия: (1) $\operatorname{brm}\left(g, \mathbb{C}^{k}\right) \geqslant k+1 \geqslant 2 ;(2)$ группа Галуа $\mathbf{G a l}\left(g, \mathbb{C}^{k}\right)$ совпадает с $\mathbf{S}(n)$; (3) $d_{k+1}=\operatorname{dim}_{\mathbb{C}} \Sigma_{g}\left(\mathbb{C}^{k}, k+1\right)=0$. В силу цитированной выше теоремы из [42] такая функция $g$ не допускает точного представления суперпозициями целых алгеброидных функций от менее чем $k$ переменных.

В. И. Арнольд полагал, что в точном представлении функции $u_{n}$ суперпозициями любых (а не только целых) алгебраических функций число необходимых переменных не может быть меньше, чем $n-2$. Теоремы 3,4 и 14 (для $X=\mathbb{C P}^{1}$ ) позволяют подтвердить одну из версий этого предположения. Но у меня не осталось места (даже на полях!), чтобы привести необходимые определения и изложить доказательство. Кое-что об этом можно найти в [48], [50, п. 11.2].

Заключительные замечания. С частью истории задач, известных под общим именем «Тринадиатая проблема Гильберта», можно познакомиться по работам Диксмье [15], Витушкина [61], [62] и Арнольда [7]. Кроме работ самого Гильберта (и тех, что уже цитировались), этой проблеме посвящены работы 
Вимана [63], Чеботарёва [55], [56], [58], Сегре [53], [54], Чеботарёва-сына [14], Морозова [52], Хованского [33]-[35], Булера и Рейхштейна [12] (ср. с попытками Н. Г. Чеботарёва «одевания» (Einkleidung) конечных групп). По-моему, после знаменитой теоремы Колмогорова-Арнольда о суперпозициях непрерывных функций самым интересным остается вопрос о наименышем числе переменных в представлении ростка однозначной ветви функции $u_{n}$ в точке, не лежащей на ее множестве ветвления, суперпозициями ростков голоморфных функций. Методы данной статьи работают либо во всем пространстве, либо в окрестности наиболее особой точки функции $u_{n}$ - начала координат.

\section{ЛитературА}

[1] В. И. Арнольд, Косы алгебраических функиий и когомологии ласточкиных хвостов, УМН, 23:4 (1968), 247-248.

[2] В. И. Арнольд, О некоторых топологических инвариантах алгебраических функиий, Труды ММО, т. 21, МГУ, М., 1970, 27-46.

[3] В. И. Арнольд, О классах когомологий алгебрачческих функиий, инвариантных относительно преобразований Чирнгаузена, Функц. анализ и его прил., 4:1 (1970), 84-85.

[4] В. И. Арнольд, Топологические инварианты алгебраических функиий. II, Функц. анализ и его прил., 4:2 (1970), 1-9.

[5] V. I. Arnold, G. Shimura, Superposition of algebraic functions, in: Mathematical Developments Arising from Hilbert's Problems, Proc. Symposia in Pure and Applied Mathematics, vol. 28, Amer. Math. Soc., Providence, RI, 1976, 45-46.

[6] В. И. Арнольд, И. Г. Петровский, топологические проблемы Гильберта и современная математика, УМН, 57:4(346) (2002), 197-207.

[7] В. И. Арнольд, От проблемы Гильберта о суперпозиииях до динамических систем, в кн.: Математические события ХХ века, Фазис, Москва, 2003, 19-51.

[8] E. Artin, Theorie der Zöpfe, Abh. Math. Sem. Univ. Hamburg, 4:1 (1925), 47-72.

[9] E. Artin, Theory of braids, Ann. of Math., 48:1 (1947), 101-126.

[10] E. Artin, Braids and permutations, Ann. of Math., 48:3 (1947), 643-649.

[11] L. Bers, H. L. Royden, Holomorphic families of injections, Acta Math., 157:3-4 (1986), 259-286.

[12] J. Buhler, Z. Reichstein, On the essential dimension of a finite group, Compositio Math., 106:2 (1997), 159-179.

[13] A. Cayley, Note sur deux formules données par M. M. Eisenstein et Hesse, J. Reine Angew. Math., 29 (1845), 54-57.

[14] Г. Н. Чеботарёв, K проблеме резольвент, Учен. зап. Казанского ун-та, 114:2 (1954), 189-193.

[15] J. Dixmier, Histoire du $13^{e}$ problème de Hilbert, in: Analyse diophantienne et géométrie algébrique, Cahiers Sém. Hist. Math., Sér. 2, vol. 3, Univ. Paris VI, Paris, 1993, 85-94.

[16] C. J. Earle, I. Kra, On holomorphic mappings between Teichmüller spaces, in: Contributions to Analysis. A collection of papers dedicated to L. Bers, Acad. Press, New York, 1974, 107-124.

[17] C. J. Earle, I. Kra, On sections of some holomorphic families of closed Riemann surfaces, Acta Math., 137:1-2 (1976), 49-79.

[18] G. Eisenstein, Über eine merkwürdige identische Gleichung, J. Reine Angew. Math., 27 (1844), 105-106 (см. также: Mathematische Werke, Band I, Chelsea, New York, $1975,1989)$.

[19] E. Fadell, L. Neuwirth, Configuration spaces, Math. Scand., 10 (1962), 111-118. 
[20] Y. Feler, Configuration spaces of tori, Atti Accad. Naz. Lincei Cl. Sci. Fis. Mat. Natur. Rend. Lincei (9) Mat. Appl., 18:2 (2007), 139-151.

[21] Y. Feler, Spaces of geometrically generic configurations, J. Eur. Math. Soc., 10:3 (2008), 601-624.

[22] L. R. Ford, Automorphic Functions, Chelsea, 1951.

[23] Е. А. Горин, В. Я. Лин, Группа кос и алгебрачческие уравнения с непрерывными коэббициентами, УМН, 24:2 (1969), 225-226.

[24] Е. А. Горин, В. Я. Лин, Алгебраические уравнения с непрерывными коэффициентами и некоторые вопросы алгебраической теории кос, Мат. сб., 78:4 (1969), 579-610.

[25] J. H. Hubbard, Sur la non-existence de sections analytiques à la courbe universelle de Teichmüller, C. R. Acad. Sci. Paris Sér. A-B, 274 (1972), A978-A979.

[26] J. H. Hubbard, Sur les sections analytiques de la courbe universelle de Teichmüller, Mem. Amer. Math. Soc., 4:166 (1976).

[27] J. H. Hubbard, Teichmüller theory and applications to geometry, topology, and dynamics. Vol. 1: Teichmüller theory, Matrix Edition, Ithaca, NY, 2006.

[28] Ш. И. Калиман, Голоморфная универсальная накрывающая пространства полиномов без кратных корней, Функц. анализ и его прил., 9:1 (1975), 71.

[29] Ш. И. Калиман, Голоморфные эндоморфизмы многообразия комплексных полиномов с дискриминантом 1, УМН, 31:1 (1976), 251-252.

[30] Ш. И. Калиман, Голоморфная универсальная накрывающая пространства полиномов без кратных корней, в сб.: Теория функций, функц. анализ и их прил., вып. 25, ХГУ, Харьков, 1977, 25-35.

[31] Ш. И. Калиман, Голоморфные эндоморфизмы комплексных камер Вейля серии $D_{n}$, УMH, 34:2 (1979), 201-202.

[32] Sh. I. Kaliman, The holomorphic universal covers of polynomials without multiple roots, Selecta Math. Soviet., 12:4 (1993), 395-405.

[33] А. Г. Хованский, О представимости алгеброидных функиий суперпозициями аналитических функиий и алгеброидных функций одной переменной, Функц. анализ и его прил., 4:2 (1970), 74-79.

[34] А. Г. Хованский, О разрешимости и неразрешимости уравнений в явном виде, УМH, 59:4 (2004), 69-146.

[35] А. Г. Хованский, Топологическая теория Галуа. Разрешимость и неразрешимость уравнений в конечном виде, МЦНМО, Москва, 2008.

[36] Ф. Клейн, Лекиии об икосаэдре и решении уравнений пятой степени, Наука, Москва, 1989.

[37] В. Я. Лин, Алгеброидные функиии и голоморфные элементы гомотопических групп комплексного многообразия, ДАН СССР, 201:1 (1971), 28-31.

[38] В. Я. Лин, О представлениях группы кос перестановками, УМН, 27:3 (1972), 192.

[39] В. Я. Лин, Алгебрачческие функиии с универсалъным дискриминантным многообразием, Функц. анализ и его прил., 6:1 (1972), 81-82.

[40] В. Я. Лин, О суперпозициях алгебраических функций, Функц. анализ и его прил., 6:3 (1972), 77-78.

[41] В. Я. Лин, Представления кос перестановками, УМН, 29:1 (1974), 173-174.

[42] В. Я. Лин, Суперпозициии алгебраических функций, Функц. анализ и его прил., 10:1 (1976), 37-45.

[43] В. Я. Лин, Кось Артина и связанные с ними группь и пространства, в кн.: Итоги науки и техники. Алгебра. Топология. Геометрия, т. 17, ВИНИТИ, М., 1979, 159-227.

[44] В. Я. Лин, Лиувиллевские накрытия комплексных пространств и аменабелъные группь, Матем. сб., 132(174):2 (1987), 202-224. 
[45] V. Ya. Lin, Around the 13th Hilbert problem for algebraic functions, in: Proc. of the Hirzebruch 65 Conf. on Algebraic Geometry (Ramat Gan, 1993), Israel Math. Conference Proc., vol. 9, Bar-Ilan Univ., Ramat Gan, 1996, 307-327.

[46] V. Ya. Lin, Braids, Permutations, Polynomials-I, Max-Planck-Institut für Math., Preprint MPI 96-118, 1996.

[47] V. Ya. Lin, M. G. Zaidenberg, Liouville and Carathéodory coverings in Riemannian and complex geometry, in: Voronezh Winter Mathematical School, Amer. Math. Soc. Transl. (2), vol. 184, Amer. Math. Soc., Providence, RI, 1998, 111-130.

[48] V. Ya. Lin, Configuration Spaces of $\mathbb{C}$ and $\mathbb{C P}^{1}$ : Some Analytic Properties, MaxPlanck-Institut für Math., Preprint MPI 96-118, 2003.

[49] V. Ya. Lin, Braids and Permutations, http://arxiv.org/abs/math/0404528v1.

[50] V. Ya. Lin, Configuration Spaces of $\mathbb{C}$ and $\mathbb{C P}^{1}$ : Some Analytic Properties, http://arxiv.org/abs/math/0403120v3.

[51] А. А. Марков, Основъ алгебраической теории кос, в кн.: Труды МИАН, т. 16, Изд-во АН СССР, М.-Л., 1945.

[52] В. В. Морозов, О некоторых вопросах проблемы резолъвент, Учен. зап. Казанск. гос. ун-та, 114:2 (1954), 173-187.

[53] B. Segre, The algebraic equations of degrees $5,9,157, \ldots$, and the arithmetics upon an algebraic variety, Ann. of Math., 46 (1945), 287-301.

[54] B. Segre, Arithmetical questions on algebraic varieties, Athlom Press, London, 1951.

[55] N. G. Tschebotaröw, Über ein algebraisches Problem von Herren Hilbert, Math. Ann., 104:1 (1931), 459-471.

[56] N. G. Tschebotaröw, Über ein algebraisches Problem von Herren Hilbert-II, Math. Ann., 105:1 (1931), 240-255.

[57] N. G. Tschebotaröw, Über das Klein-Hilbertsche Resolventenproblem, Изв. Казанск. физ.-мат. общ., 6 (1932-1933), 5-22.

[58] Н. Г. Чеботарёв, Проблема резолъвент и критические многообразия, Изв. АН CССР, сер. матем.; Собр. соч., т. 1, стр. 305-326, 7 (1943), 123-146.

[59] В. А. Васильев, Когомологии группь кос и сложность алгоритмов, Функц. анализ и его прил., 22:3 (1988), 15-24.

[60] В. А. Васильев, Топология дополнений к дискриминантам, Фазис, М., 1997.

[61] A. G. Vitushkin, On representation of functions by means of superpositions and related topics, Monographie No. 25 de l'Enseignement Mathématique. Série des Conférences de l'Union Mathe'matique Internationale, No. 6, Enseignement Mathématique Université de Genéve, Genéve, 1978.

[62] А. Г. Витушкин, 13-я проблема Гилъберта и смежснье вопросъ, УМН, 59:1 (2004), 11-24.

[63] A. Wiman, Über die Anwendung der Tschirnhausentransformation auf die Reduktion algebraischer Gleichungen, Nova Acta R. Soc. Sci. Uppsalientis, vol. extra ordin. editum, 1927, 3-8.

[64] В. М. Зинде, Аналитические свойства пространств регулярнъх орбит групп Коксетера серий $B$ и D, Функц. анализ и его прил., 11:1 (1977), 69-70.

[65] В. М. Зинде, Голоморфные отображения пространств регулярных орбит групп Коксетера серий $B$ u D, Сиб. мат. журнал, 18:5 (1978), 1015-1026. 